\title{
Sleep disorders in patients with ADHD: impact and management challenges
}

This article was published in the following Dove Press journal:

Nature and Science of Sleep

\author{
Dafna Wajszilber ${ }^{1}$ \\ José Arturo Santiseban ${ }^{1,2}$ \\ Reut Gruber ${ }^{1,2}$ \\ 'Department of Psychiatry, Faculty of \\ Medicine, McGill University, Montréal, \\ Quebec, Canada; ${ }^{2}$ Attention Behavior \\ and Sleep Lab, Douglas Research \\ Center, Montréal, Quebec, Canada
}

\begin{abstract}
Attention deficit/hyperactivity disorder (ADHD) is one of the most commonly diagnosed disorders in childhood, enduring through adolescence and adulthood and presenting with symptoms of inattention, hyperactivity, and/or impulsivity and significantly impairing functioning. Primary sleep disorders such as sleep-disordered breathing, restless leg syndrome, circadian rhythm sleep disorder, insomnia, and narcolepsy are commonly comorbid in these individuals but not often assessed and are therefore often left untreated. Sleep disturbances in individuals with ADHD can result in significant functional impairments that affect mood, attention, behavior, and ultimately school/work performance and quality of life. Previous reviews have described findings related to sleep but have neglected to examine potential impacts of these sleep disorders and ADHD on daytime functioning. This review investigates empirical findings pertaining to sleep abnormalities and related cognitive, behavioral, emotional, and physical impairments in individuals with ADHD and comorbid primary sleep disorders across the life span. It discusses implications to management and highlights existing limitations and recommended future directions.
\end{abstract}

Keywords: ADHD, impairments, sleep-disordered breathing, restless leg syndrome, circadian rhythm sleep disorders, insomnia, narcolepsy

\section{Introduction}

Attention-deficit/hyperactivity disorder (ADHD) is one of the most commonly diagnosed disorders in childhood and adulthood. It affects approximately $3 \%-5 \%$ of youth ${ }^{1}$ and $2.9 \%$ of adults. ${ }^{2}$ It endures throughout adolescence and adulthood. A diagnosis of ADHD is dependent on developmentally inappropriate symptoms of inattention, hyperactivity, and/or impulsivity, with onset before the age of 12 years and impaired functioning in two or more settings. If untreated, individuals with ADHD struggle with impairments across many crucial domains of functioning, including academic, occupational, and social realms. ${ }^{3}$

In clinical practice, sleep problems are reported in an estimated $25 \%-50 \%$ of individuals who have ADHD, ${ }^{4-6}$ and adults who do not get the recommended amount of sleep are more likely to report ADHD symptoms. ${ }^{7}$ Sleep disturbances in such individuals have been associated with comorbid primary sleep issues and/or alterations associated with the medications used to treat ADHD. ${ }^{8}$ In an affected individual, sleep disturbances that result in sleep restriction or sleep fragmentation can lead to excessive daytime fatigue and interfere with mood, attention, behavior, and physical health, all of which are critical for school/work performance and a good quality of life. However, although sleep problems are very common in individuals with ADHD, comorbid sleep
Correspondence: Reut Gruber Department of Psychiatry, Faculty of Medicine, McGill University, 6875 Boulevard LaSalle, Perry Pavilion, E-410, Montréal, QC H4H IR3, Canada Tel + 5 I4 76I 6I3I; (ext. 3476) Email reut.gruber@douglas.mcgill.ca 
disorders are often overlooked and left untreated in ADHD populations.

Researchers have examined the associations between ADHD and sleep-disordered breathing (SDB), restless leg syndrome (RLS), circadian rhythm sleep disorders (CRSDs), insomnia, and narcolepsy. ${ }^{9-11} \mathrm{SDB}$ is characterized by abnormal patterns of respiration and/or ventilation during sleep, ${ }^{12}$ while obstructive sleep apnea (OSA) is characterized by partial or complete upper airway obstruction, leading to disrupted sleep. ${ }^{13,14} \mathrm{~A}$ history of snoring or possible OSA during childhood is associated with a twofold difference in the odds of ADHD diagnosis or symptoms, ${ }^{15,16}$ and elevated incidence of SDB is found among individuals with ADHD. ${ }^{17-21}$ SDB affects psychological outcomes through various mechanisms, including the deleterious effects of hypoxic insults and the resulting stress/inflammation in the brain and/or repeated arousal-based sleep disruptions. ${ }^{22}$ These mechanisms can alter the neurochemical substrates of the prefrontal cortex ${ }^{23}$ leading to neurobehavioral deficits underlying ADHD symptoms.

RLS is a common sensorimotor disorder characterized by an irresistible urge to move the legs, often accompanied by uncomfortable sensations in the legs or (less frequently) other body parts. These sensations are worse at rest, relieved by movement, and most bothersome in the evening or night. Patients frequently experience insomnia from leg discomfort and the need to move around. Dopaminergic abnormalities and iron deficiency are presumed to underlie this comorbidity. ${ }^{24-31}$ Although the prevalence of RLS in the pediatric population is unknown, approximately $10 \%$ of adults in the USA have the disorder. The evidence suggests that up to $44 \%$ of individuals with ADHD have RLS or RLS-like symptoms and up to $26 \%$ of individuals who have RLS have ADHD or ADHD-like symptoms. ${ }^{28,30,32-35}$ The underlying pathophysiology linking RLS and ADHD may lie in the deficit of dopamine in the nigrostriatal brain region, which has been associated with both disorders. ${ }^{36}$ In addition, iron deficiency has also been linked to ADHD and RLS,${ }^{37}$ since iron is a cofactor for tyrosine hydroxylase, the rate-limiting enzyme responsible for dopamine synthesis, which also influences the dopamine pathway directly. Sleep disruption itself may lead to symptoms of ADHD, and daytime symptoms of RLS such as restlessness and inattention may mimic ADHD symptoms. ${ }^{38}$

CRSDs involve a problem in the timing when a person sleeps and is awake. They are caused by alterations of the circadian time-keeping system or its entrainment mechanisms or by a misalignment of the endogenous circadian rhythm and the external environment. Delayed sleep phase syndrome (DSPS) occurs when a person regularly goes to sleep and wakes up $>2$ hours later than is considered normal. Individuals with ADHD have been found to have changes in these mechanisms, decreases in the volume of the pineal gland, and/or clock gene abnormalities; in particular, a recent study conducted by Baird et al found that the expression of clock genes BMAL1 and PER2 was altered in individuals with ADHD. ${ }^{39}$ DSPS and late chronotype are frequently comorbid in adults ${ }^{3,40,41}$ and adolescents ${ }^{42}$ with ADHD. The pathophysiology linking ADHD and CRSD maybe both behavioral and biological. ${ }^{43}$ Deficits in impulsivity control may affect the ability of an individual to settle down, leading to bedtime resistance and resulting in delayed sleep onset. In addition, it is proposed that individuals with ADHD may have a stronger circadian evening preference and possible endogenous melatonin increase delay. ${ }^{43,44}$

Insomnia is a sleep disorder characterized by difficulty falling or staying asleep, even when a person has the chance to do so. ${ }^{45}$ Prolonged sleep onset, delayed bedtime, and frequent night awakenings are commonly seen in individuals with ADHD. It is more frequent among children $(73.3 \%)^{46}$ and adults with ADHD $(66.8 \%)^{47}$ than in the general population (children: $20 \%-30 \%{ }^{48}$ adults: $6 \%-50 \%{ }^{49,50}$ ). Unhealthy sleep practices and a lack of routines, especially at bedtime, have been shown to be more common in individuals with ADHD and to have a significant negative impact on sleep initiation. ${ }^{51}$ In addition, these sleep issues could result from increased resistance at bedtime, difficulty in settling down in the evening, or poor sleep hygiene.

Narcolepsy is a chronic neurological disease that manifests as difficulty with maintaining continuous wake and sleep. The clinical presentation varies, but a diagnosis of narcolepsy requires excessive daytime sleepiness (EDS) occurring alone or together with features of rapid eye movement (REM), sleep dissociation (eg, cataplexy, hypnagogic/ hypnopompic hallucinations, sleep paralysis), and disrupted nighttime sleep. There are two major types of narcolepsy: type 1 narcolepsy diagnosis is based on the individual either having low levels of hypocretin or reporting cataplexy and having EDS; type 2 narcolepsy diagnosis involves EDS in the absence of cataplexy and with normal hypocretin levels. ${ }^{45}$ Retrospectively, adults with narcolepsy were found to have a twofold greater likelihood of having a childhood diagnosis of ADHD compared with controls. ${ }^{52}$ In addition, evidence suggests that children with ADHD experience hypoarousal and that their hyperactive/impulsive symptoms may be compensatory behaviors for fatigue. ${ }^{53}$ The link between ADHD 
and narcolepsy is poorly understood, ${ }^{11}$ but it is hypothesized that EDS in individuals with narcolepsy could result in inattention, poor executive function, and impulse control problems that mimic ADHD and respond well to stimulant medication. ${ }^{54-56}$ Ultimately, the overlap of symptoms of ADHD and narcolepsy may lead to diagnostic confusion or misdiagnosis of the disorders. ${ }^{54}$ Another hypothesis is that the link may lie in a shared brain pathophysiology. ${ }^{57}$ In ADHD, a suspected dysregulation of dopamine and noradrenaline is an accepted hypothesis, ${ }^{58}$ and REM sleep, in turn, is affected by noradrenergic and cholinergic neurons. ${ }^{59}$ Dysregulation of noradrenaline could lead to changes in REM sleep similar to that seen in patients with narcolepsy. ${ }^{57}$ In addition, medication geared at treating ADHD targets the abovementioned neurotransmitters, ${ }^{60}$ and there is an overlap with medication used to treat narcolepsy. ${ }^{61,62}$ There may also be a possible genetic link between narcolepsy and ADHD. Individuals with ADHD and hypersomnia can have shorter REM latencies and fulfill the criteria for type 2 narcolepsy. ${ }^{57}$ In addition, these individuals tend to have an absence of the DQB $1 * 06: 02$ allele that is usually found in individuals with type 1 narcolepsy and some individuals with type 2 narcolepsy without ADHD, alluding to the possibility that individuals with ADHD and hypersomnia could be part of a subtype of type 2 narcolepsy. ${ }^{57}$

Previous reviews have described research findings pertaining to sleep and the mechanisms that may underlie the comorbidity between the abovedescribed primary sleep disorders and ADHD. ${ }^{63}$ However, the existing reviews have neglected to examine the potential impacts of these disorders on the daytime functioning of individuals who present with ADHD and sleep issues. ${ }^{64-66}$ This is a problem because in order to provide optimal care, clinicians need to be aware of sleep problems among patients with ADHD and their potential impacts on the very symptoms they are attempting to treat and manage. An investigation of how sleep disturbances impact ADHD symptoms will help clarify the outcomes and management challenges associated with treating individuals with ADHD and comorbid primary sleep disorders. Hence, the goal of the present review is to describe empirical findings pertaining to sleep abnormalities and cognitive, behavioral, emotional, or physical outcomes associated with sleep issues in individuals with ADHD and comorbid primary sleep disorders.

\section{Methods}

We employed a narrative review methodology to identify and synthesize empirical studies that have examined the sleep patterns of individuals with ADHD and each of the primary sleep disorders known to be comorbid with ADHD, namely SDB, RLS, CRSD, insomnia, and narcolepsy. Three electronic databases were searched in July 2018 (Embase, MEDLINE, and PsycINFO) for studies published in the prior 5 years (between January 1, 2013 and July 1, 2018). Additional records were identified by searching the references of the selected original research papers and review articles.

Separate parallel searches were conducted for each primary sleep disorder using the following key words: ADHD and narcolepsy; ADHD and CRSD, dim light melatonin onset, light therapy, or melatonin; ADHD and restless leg syndrome, RLS, periodic and extremities, periodic limb movement, iron, ferritin, ferritins, levodopa, or L-DOPA; ADHD and sleep apnea or sleep-disordered breathing; and ADHD and insomnia. The inclusion criteria were: 1) peer-reviewed original research of subjects with a sleep disorder or ADHD, in which variables related to both ADHD and sleep were measured; and 2) studies that were written in the English language and used quantitative methods. All the included published empirical studies are listed in the summary table (see Table 1). In total, the literature search yielded 39 relevant papers, including thirteen, seven, eight, seven, and six for ADHD and SDB, RLS, CRSDs, insomnia, and narcolepsy, respectively.

\section{Results}

In the following sections, findings based on recent empirical data on the sleep characteristics and their associations with daytime functioning and physical health of individuals with ADHD primary sleep disorders are reviewed. These results are organized separately for each of these subgroups in the following way: findings regarding sleep, sleepiness, or circadian characteristics are subdivided according the nature of the tools that were used (objective or subjective measures) and the age groups of the participants. Daytime impairments are divided into cognitive, emotional, and physical outcomes. Detailed information for each study referenced can be found in Table 1 .

\section{ADHD and SDB}

The present review includes 13 studies (six cross-sectional, two case-control, one retrospective cohort, two prospective cohort, and two open-label) that examined the sleep characteristics of individuals with SDB and ADHD.

\section{Sleep characteristics ${ }^{67-76}$}

Polysomnography (PSG)

School-age children. N/A; Adolescents. N/A; Adults. No significant differences in apnea-hypopnea index, a measure 
Table I Summary of studies reviewed

\begin{tabular}{|c|c|c|c|c|}
\hline Author and year & Sample & Study design & Diagnoses & Measures \\
\hline \multicolumn{5}{|c|}{ Table I.I OSA and sleep-disordered breathing } \\
\hline Smith et al $(2017)^{67}$ & $\begin{array}{l}\text { 63I children } \\
\text { Sex: } 347 \text { males, } 284 \text { females } \\
\text { Age (mean } \pm \text { SD), years: } \\
6.94 \pm 1.30\end{array}$ & Cross-sectional & $\begin{array}{l}\text { ADHD: CPRS-R, } \\
\text { CBCL } \\
\text { OSA/SDB: PSG }\end{array}$ & $\begin{array}{l}\text { Sleep: PSG } \\
\text { Attention: CPRS-R, } \\
\text { CBCL } \\
\text { Cognitive: NEPSY, } \\
\text { NEPSY-II } \\
\text { Intelligence: DAS }\end{array}$ \\
\hline Kim et al $(2018)^{68}$ & $\begin{array}{l}170 \text { children with AT, I50 } \\
\text { healthy controls } \\
\text { Sex: I } 82 \text { males, I } 35 \text { females } \\
\text { Age (mean } \pm \text { SD), years: OSA/ } \\
\text { SDB: } 7.7 \pm I .5 \text {; controls: } 7.6 \pm 1.5\end{array}$ & $\begin{array}{l}\text { Prospective cohort } \\
\text { intervention: AT }\end{array}$ & $\begin{array}{l}\text { ADHD: SDQ } \\
\text { OSA/SDB: PSG, } \\
\text { SRBD }\end{array}$ & $\begin{array}{l}\text { Sleep: PSG, SRBD } \\
\text { Attention: SDQ } \\
\text { Socioemotional: } \\
\text { CDI } \\
\text { SCARED } \\
\text { Physical: BMI }\end{array}$ \\
\hline Wu et al $(2017)^{80}$ & $\begin{array}{l}437 \text { children with OSA } \\
\text { Sex: } 298 \text { males, } 139 \text { females } \\
\text { Age (mean } \pm \text { SD), years: } \\
5.71 \pm 1.45\end{array}$ & Retrospective cohort & $\begin{array}{l}\text { ADHD: DSM-IV } \\
\text { criteria } \\
\text { OSA/SDB: PSG }\end{array}$ & $\begin{array}{l}\text { Sleep: OSA-18 } \\
\text { Socioemotional: } \\
\text { OSA-18 } \\
\text { Physical: OSA-18 }\end{array}$ \\
\hline Smith et al $(2016)^{69}$ & $\begin{array}{l}\text { I,022 children } \\
\text { Sex: } 562 \text { males, } 460 \text { females } \\
\text { Age (mean } \pm S D \text { ), years: } \\
6.86 \pm 0.89\end{array}$ & Cross-sectional & $\begin{array}{l}\text { ADHD: CPRS-R, } \\
\text { CBCL } \\
\text { OSA/SDB: PSG } \\
\text { Parental report }\end{array}$ & $\begin{array}{l}\text { Sleep: PSG } \\
\text { Attention: CPRS-R, } \\
\text { CBCL }\end{array}$ \\
\hline Smith et al $(2017)^{67}$ & $\begin{array}{l}\text { I, I I6 children } \\
\text { Sex: } 598 \text { males, } 518 \text { females } \\
\text { Age (mean } \pm \text { SD), years: } \\
6.84 \pm 0.86\end{array}$ & Cross-sectional & $\begin{array}{l}\text { ADHD: CPRS-R } \\
\text { OSA/SDB: PSG }\end{array}$ & $\begin{array}{l}\text { Sleep: PSG } \\
\text { Attention: CPRS-R, } \\
\text { CBCL, NEPSY, } \\
\text { NEPSY-II }\end{array}$ \\
\hline Villa et al $(2016)^{71}$ & $\begin{array}{l}76 \text { children with suspected OSA } \\
\text { Sex: } 44 \text { males, } 32 \text { females } \\
\text { Age (mean } \pm \text { SD), years: } \\
4.95 \pm 1.75\end{array}$ & $\begin{array}{l}\text { Open-label trial } \\
\text { intervention: AT, } \\
\text { RME, or medical } \\
\text { therapy }\end{array}$ & $\begin{array}{l}\text { ADHD: DSM-IV } \\
\text { criteria } \\
\text { OSA/SDB: PSG } \\
\text { Medical history } \\
\end{array}$ & \begin{tabular}{|l|} 
Sleep: PSG \\
Attention: \\
unspecified DSM-IV \\
symptom checklist \\
\end{tabular} \\
\hline Zhu et al $(2014)^{72}$ & $\begin{array}{l}\text { 5I children with OSA } \\
\text { Sex: } 35 \text { males, } 16 \text { females } \\
\text { Age (mean } \pm \text { SD), years: } \\
7.68 \pm 2.15\end{array}$ & $\begin{array}{l}\text { Open-label trial } \\
\text { intervention: AT }\end{array}$ & $\begin{array}{l}\text { ADHD: IVA-CPT } \\
\text { OSA/SDB: PSG }\end{array}$ & $\begin{array}{l}\text { Sleep: PSG } \\
\text { Attention: IVA-CPT }\end{array}$ \\
\hline
\end{tabular}




\begin{tabular}{|c|c|c|c|c|}
\hline \multirow[t]{2}{*}{ Sleep differences } & \multicolumn{3}{|l|}{ Daytime impairments } & \multirow[t]{2}{*}{ Conclusions } \\
\hline & Cognitive & Emotional & Physical & \\
\hline N/A & $\begin{array}{l}\text { Snorers vs nonsnorers: more } \\
\text { ADHD symptoms. } \\
\text { Higher AHI was associated } \\
\text { with less hyperactivity and } \\
\text { inattention symptoms. } \\
\text { Increased snoring, but not } \\
\text { AHI, was associated with } \\
\text { worse performance on } \\
\text { cognitive tests. }\end{array}$ & $\begin{array}{l}\text { Snorers vs } \\
\text { nonsnorers: } \\
\text { more } \\
\text { internalizing } \\
\text { and } \\
\text { externalizing } \\
\text { problems. }\end{array}$ & N/A & $\begin{array}{l}\text { Snoring, but not } \mathrm{AHI} \text {, } \\
\text { was associated with more } \\
\text { daytime dysfunction in } \\
\text { attention, cognition, and } \\
\text { psychological aspects. }\end{array}$ \\
\hline AT improved SDB symptoms & $\begin{array}{l}\text { AT reduced hyperactivity and } \\
\text { inattention symptoms. } \\
\text { This improvement was } \\
\text { reduced in older children (vs } \\
\text { younger) and obese children } \\
\text { (vs normal weight). }\end{array}$ & $\begin{array}{l}\text { AT reduced } \\
\text { symptoms of } \\
\text { anxiety and } \\
\text { depression. }\end{array}$ & $\begin{array}{l}\text { AT } \\
\text { increased } \\
\text { odds of } \\
\text { being } \\
\text { overweight. }\end{array}$ & $\begin{array}{l}\text { AT improved symptoms } \\
\text { of SDB and daytime } \\
\text { symptoms of ADHD, } \\
\text { depression, and anxiety. } \\
\text { Young children with } \\
\text { normal weight had better } \\
\text { improvement. }\end{array}$ \\
\hline $\begin{array}{l}\text { OSA + ADHD vs OSA: more } \\
\text { sleep disturbances, higher AHI, } \\
\text { and lower oxygen saturation. }\end{array}$ & $\begin{array}{l}\text { OSA + ADHD vs OSA: more } \\
\text { daytime problems. }\end{array}$ & $\begin{array}{l}\text { OSA + } \\
\text { ADHD vs } \\
\text { OSA: greater } \\
\text { emotional } \\
\text { distress and } \\
\text { caretaker } \\
\text { concern. }\end{array}$ & $\begin{array}{l}\text { OSA + } \\
\text { ADHD vs } \\
\text { OSA: more } \\
\text { physical } \\
\text { suffering }\end{array}$ & $\begin{array}{l}\text { OSA with comorbid } \\
\text { ADHD had worse } \\
\text { daytime functioning across } \\
\text { cognitive, emotional, } \\
\text { and physical outcomes } \\
\text { compared with OSA } \\
\text { without ADHD. }\end{array}$ \\
\hline N/A & $\begin{array}{l}\text { Snorers with normal AHI vs } \\
\text { nonsnorers with normal AHI: } \\
\text { worse CPRS-R. } \\
\text { Snorers with normal AHI vs } \\
\text { nonsnorers with normal AHI, } \\
\text { mild AHI, severe AHI: higher } \\
\text { hyperactivity and ADHD } \\
\text { scores on the CBCL. }\end{array}$ & $\begin{array}{l}\text { Snorer with } \\
\text { normal AHI } \\
\text { vs nonsnorers } \\
\text { with normal } \\
\text { AHI: worse } \\
\text { internalizing. }\end{array}$ & N/A & $\begin{array}{l}\text { Snoring with a normal } \mathrm{AHI} \\
\text { is associated with worse } \\
\text { daytime functioning for } \\
\text { ADHD and internalizing } \\
\text { than either snorers } \\
\text { with abnormal AHI or } \\
\text { nonsnorers with normal } \\
\text { AHI. }\end{array}$ \\
\hline $\mathrm{N} / \mathrm{A}$ & $\begin{array}{l}\text { SDB is associated with } \\
\text { behavioral problems. SDB } \\
\text { was not directly associated } \\
\text { with cognitive problems. } \\
\text { However, behavioral } \\
\text { problems were an indirect } \\
\text { mediator of the association } \\
\text { between SDB and cognitive } \\
\text { problems. }\end{array}$ & $\mathrm{N} / \mathrm{A}$ & $\mathrm{N} / \mathrm{A}$ & $\begin{array}{l}\text { SDB is associated with } \\
\text { more behavioral problems, } \\
\text { which may lead to cognitive } \\
\text { problems. }\end{array}$ \\
\hline $\begin{array}{l}\text { AHI improved after AT and } \\
\text { RME. } \\
\text { Mean } \mathrm{O}_{2} \text { saturation improved in } \\
\text { AT group. }\end{array}$ & $\begin{array}{l}\text { AT improved ADHD } \\
\text { symptoms. }\end{array}$ & $\mathrm{N} / \mathrm{A}$ & $\mathrm{N} / \mathrm{A}$ & $\begin{array}{l}\text { AT was better at improving } \\
\text { symptoms of ADHD than } \\
\text { RME or medical therapy. }\end{array}$ \\
\hline $\begin{array}{l}\mathrm{AHI} \text { and mean } \mathrm{O}_{2} \text { saturation } \\
\text { improved between baseline and } \\
3 \text { months follow-up, as well } \\
\text { as between } 3 \text { and } 6 \text { months } \\
\text { follow-up. } \\
\text { Those with severe OSA had } \\
\text { worse parameters at baseline, } \\
\text { but at } 6 \text { months follow-up there } \\
\text { were no differences based on } \\
\text { initial severity. }\end{array}$ & $\begin{array}{l}\text { IVA-CPT scores improved } \\
\text { from baseline to } 3 \text { months } \\
\text { follow-up, as well as from } 3 \\
\text { to } 6 \text { months follow-up. } \\
\text { Severity of OSAHS was } \\
\text { associated with worse Full } \\
\text { Attention Quotient scores, } \\
\text { but not Hyperactivity or } \\
\text { Full Scale Response Control } \\
\text { Quotient. }\end{array}$ & N/A & $\mathrm{N} / \mathrm{A}$ & $\begin{array}{l}\text { AT improved performance } \\
\text { on a cognitive test of } \\
\text { attention. Performance } \\
\text { continued to improve } \\
\text { until at least } 6 \text { months } \\
\text { follow-up. }\end{array}$ \\
\hline
\end{tabular}

(Continued) 
Table I (Continued)

\begin{tabular}{|c|c|c|c|c|}
\hline Author and year & Sample & Study design & Diagnoses & Measures \\
\hline Vitelli et al $(2015)^{73}$ & $\begin{array}{l}36 \text { children with OSA, } 38 \\
\text { children with OSA + obesity, } 58 \\
\text { controls } \\
\text { Sex: } 77 \text { males, } 57 \text { females } \\
\text { Age (mean } \pm \text { SD), years: OSA: } \\
8.29 \pm 2.04 \text {; OSA + obesity: } \\
8.73 \pm 1.69 \text {; controls: } 8.89 \pm 1.62\end{array}$ & Case-control & $\begin{array}{l}\text { ADHD: ADHD-RS } \\
\text { OSA/SDB: PSG }\end{array}$ & $\begin{array}{l}\text { Sleep: PSG } \\
\text { Attention: ADHD- } \\
\text { RS } \\
\text { Physical: BMI }\end{array}$ \\
\hline $\begin{array}{l}\text { Oğuztürk et al } \\
(20 \mid 3)^{74}\end{array}$ & $\begin{array}{l}81 \text { treatment-naïve adults with } \\
\text { OSA, } 32 \text { controls } \\
\text { Sex: } 89 \text { males, } 27 \text { females } \\
\text { Age (mean } \pm \text { SD), years: OSA: } \\
48 . I \pm 8.9 \text {; controls: } 44 . I \pm 13.2\end{array}$ & Case-control & $\begin{array}{l}\text { ADHD: ASRS } \\
\text { OSA/SDB: PSG }\end{array}$ & $\begin{array}{l}\text { Sleep: PSG } \\
\text { Sleepiness: ESS } \\
\text { Attention: ASRS } \\
\text { Socioemotional: } \\
\text { HADS } \\
\text { Physical: SF-36 }\end{array}$ \\
\hline $\begin{array}{l}\text { Perfect et al } \\
(20 \mid 3)^{75}\end{array}$ & $\begin{array}{l}263 \text { children } \\
\text { Sex: I } 23 \text { males, } 140 \text { females } \\
\text { Age (mean } \pm \text { SD), years: } \\
13.22 \pm 1.70\end{array}$ & Prospective cohort & $\begin{array}{l}\text { ADHD: medical } \\
\text { history } \\
\text { OSA/SDB: PSG } \\
\text { Parent-reported } \\
\text { snoring }\end{array}$ & \begin{tabular}{|l} 
Sleep: PSG \\
SHQ \\
Attention: BASC-2 \\
Socioemotional: \\
ABAS-II \\
School problems \\
parent report \\
\end{tabular} \\
\hline Ekici et al $(2013)^{76}$ & $\begin{array}{l}90 \text { adult snorers or with OSA } \\
\text { Sex: } 67 \text { males, } 23 \text { females } \\
\text { Age (mean } \pm S D \text { ), years: } \\
44.23 \pm 10.23\end{array}$ & Cross-sectional & $\begin{array}{l}\text { ADHD: ASRS } \\
\text { OSA/SDB: PSG }\end{array}$ & $\begin{array}{l}\text { Sleep: PSG } \\
\text { Attention: ASRS }\end{array}$ \\
\hline Amiri et al $(2015)^{78}$ & $\begin{array}{l}53 \text { post-AT children with } \\
\text { untreated ADHD } \\
\text { Sex: } 28 \text { males, } 25 \text { females } \\
\text { Age (mean } \pm \text { SD), years: } \\
6.89 \pm 2.06\end{array}$ & $\begin{array}{l}\text { Prospective cohort } \\
\text { intervention: AT }\end{array}$ & $\begin{array}{l}\text { ADHD: K-SADS-PL } \\
\text { OSA/SDB: direct } \\
\text { observation of } \\
\text { tonsils and medical } \\
\text { history }\end{array}$ & Attention: CPRS-R \\
\hline \multicolumn{5}{|c|}{ Table I.2 RLS and periodic limb movement disorder } \\
\hline Roy et al $(20 \mid 8)^{86}$ & $\begin{array}{l}\text { I,632 adults } \\
\text { Sex: } 757 \text { males, } 875 \text { females } \\
\text { Age (mean } \pm \text { SD), years: } \\
43.2 \pm I 2.7\end{array}$ & Cross-sectional & $\begin{array}{l}\text { ADHD: ADHD-RS } \\
\text { RLS: IRLSSG } \\
\text { criteria }\end{array}$ & $\begin{array}{l}\text { Sleep: JSS } \\
\text { Attention: ADHD- } \\
\text { RS, WURS-K }\end{array}$ \\
\hline Um et al $(2016)^{82}$ & $\begin{array}{l}28 \text { children with ADHD } \\
\text { Sex: } 22 \text { males, } 6 \text { females } \\
\text { Age (mean } \pm \text { SD), years: male: } \\
8.95 \pm 2.26 \text {; female: } 9.17 \pm 2.04\end{array}$ & Cross-sectional & $\begin{array}{l}\text { ADHD: DSM-IV-TR } \\
\text { criteria } \\
\text { PLMD: PSG }\end{array}$ & $\begin{array}{l}\text { Sleep: PSG } \\
\text { Attention: CGI-P } \\
\text { Cognitive: MFFT- } \\
\text { KC } \\
\text { Intelligence: } \\
\text { K-WISC-III }\end{array}$ \\
\hline
\end{tabular}




\begin{tabular}{|c|c|c|c|c|}
\hline \multirow[t]{2}{*}{ Sleep differences } & \multicolumn{3}{|l|}{ Daytime impairments } & \multirow[t]{2}{*}{ Conclusions } \\
\hline & Cognitive & Emotional & Physical & \\
\hline N/A & $\begin{array}{l}\text { OSA + Obesity vs OSA: } \\
\text { more symptoms of ADHD. }\end{array}$ & $\mathrm{N} / \mathrm{A}$ & $\mathrm{N} / \mathrm{A}$ & $\begin{array}{l}\text { In children with OSA, } \\
\text { obesity is associated with } \\
\text { greater symptoms of } \\
\text { ADHD (vs normal weight). }\end{array}$ \\
\hline $\begin{array}{l}\text { OSA + ADHD vs OSA: higher } \\
\text { sleepiness, no PSG differences. }\end{array}$ & $\begin{array}{l}\text { OSA vs controls: no } \\
\text { differences in ADHD } \\
\text { prevalence. }\end{array}$ & $\begin{array}{l}\text { OSA + ADHD } \\
\text { vs OSA: more } \\
\text { anxiety and } \\
\text { depression } \\
\text { symptoms. }\end{array}$ & $\begin{array}{l}\text { OSA + } \\
\text { ADHD vs } \\
\text { OSA: lower } \\
\text { physical } \\
\text { health } \\
\text { quality of } \\
\text { life, no } \\
\text { difference } \\
\text { in BMI } \\
\end{array}$ & $\begin{array}{l}\text { OSA with comorbid } \\
\text { ADHD has worse } \\
\text { emotional and physical } \\
\text { outcomes than OSA } \\
\text { without ADHD. }\end{array}$ \\
\hline $\mathrm{N} / \mathrm{A}$ & $\begin{array}{l}\text { Persistent SDB vs never SDB: } \\
\text { no significant differences in } \\
\text { ADHD diagnosis, greater } \\
\text { hyperactivity, and lower } \\
\text { school grades. }\end{array}$ & $\begin{array}{l}\text { Persistent } \\
\text { SDB vs never } \\
\text { SDB: impaired } \\
\text { social and } \\
\text { communication } \\
\text { skills. }\end{array}$ & $\mathrm{N} / \mathrm{A}$ & $\begin{array}{l}\text { Presence of current SDB } \\
\text { and persistence of SDB are } \\
\text { associated with increased } \\
\text { hyperactivity symptoms, } \\
\text { impaired social skills, and } \\
\text { lower school grades. }\end{array}$ \\
\hline $\mathrm{N} / \mathrm{A}$ & $\begin{array}{l}\text { Snoring vs OSA: no } \\
\text { difference in ADHD } \\
\text { symptoms. }\end{array}$ & $\mathrm{N} / \mathrm{A}$ & $\mathrm{N} / \mathrm{A}$ & $\begin{array}{l}\text { No difference was found } \\
\text { in cognitive outcomes } \\
\text { between snorers and OSA } \\
\text { adults. }\end{array}$ \\
\hline $\mathrm{N} / \mathrm{A}$ & $\begin{array}{l}\text { AT improved ADHD } \\
\text { symptoms between baseline } \\
\text { and } 3 \text { months follow-up, as } \\
\text { well as between } 3 \text { months } \\
\text { follow-up and } 6 \text { months } \\
\text { follow-up. }\end{array}$ & $\mathrm{N} / \mathrm{A}$ & $\mathrm{N} / \mathrm{A}$ & $\begin{array}{l}\text { AT improved symptoms } \\
\text { of ADHD and symptoms } \\
\text { continue to improve at } \\
\text { least until } 6 \text { months after } \\
\text { surgery. }\end{array}$ \\
\hline $\begin{array}{l}\text { Adult ADHD is associated } \\
\text { with increased odds of } \\
\text { presenter RLS, OR }=4.73 \text {. } \\
\text { After controlling for sleep } \\
\text { disturbances, this association } \\
\text { was no longer significant, which } \\
\text { suggests that sleep disturbances } \\
\text { may partially mediate the } \\
\text { relationship between ADHD } \\
\text { and RLS. }\end{array}$ & $\begin{array}{l}\text { RLS vs non-RLS: prevalence } \\
\text { of ADHD is } 17.3 \% \text { vs } 4.2 \% \text {. }\end{array}$ & $\mathrm{N} / \mathrm{A}$ & $\mathrm{N} / \mathrm{A}$ & $\begin{array}{l}\text { The prevalence of RLS } \\
\text { is higher in adults with } \\
\text { ADHD. }\end{array}$ \\
\hline $\begin{array}{l}\text { Limb movement index: } \\
\text { I I.64 } \pm 3.90 \text {. Limb movement } \\
\text { index with arousal: } 6.74 \pm 2.33 \text {. } \\
\text { Periodic limb movement: } \\
0.30 \pm 1.57 \text {. Periodic limb } \\
\text { movement with arousal: } \\
0.05 \pm 0.25 \text {. }\end{array}$ & $\begin{array}{l}\text { Higher limb movement index } \\
\text { with arousal was associated } \\
\text { with worse verbal IQ and } \\
\text { number of correct answers in } \\
\text { the MFFT-KC. }\end{array}$ & N/A & $\mathrm{N} / \mathrm{A}$ & $\begin{array}{l}\text { In children with ADHD, } \\
\text { more symptoms of PLMD } \\
\text { are associated with } \\
\text { reduced performance on } \\
\text { intelligence and cognitive } \\
\text { tests. }\end{array}$ \\
\hline
\end{tabular}

(Continued) 
Table I (Continued)

\begin{tabular}{|c|c|c|c|c|}
\hline Author and year & Sample & Study design & Diagnoses & Measures \\
\hline $\begin{array}{l}\text { Ghorayeb et al } \\
(2017)^{87}\end{array}$ & $\begin{array}{l}105 \text { adults with RLS } \\
\text { Sex: } 43 \text { males, } 62 \text { females } \\
\text { Age (mean } \pm \text { SD), years: } \\
41.75 \pm 19.2\end{array}$ & Cross-sectional & $\begin{array}{l}\text { ADHD: ASRS } \\
\text { RLS: IRLSSG }\end{array}$ & $\begin{array}{l}\text { RLS: serum ferritin } \\
\text { and iron. } \\
\text { Attention: ASRS } \\
\text { Socioemotional: } \\
\mathrm{MOCl}\end{array}$ \\
\hline Ferri et al $(2013)^{83}$ & $\begin{array}{l}18 \text { children with ADHD, } 17 \\
\text { health controls } \\
\text { Sex: } 20 \text { males, I5 females } \\
\text { Age (mean } \pm \text { SD), years: } \\
\text { ADHD: } 8.9 \pm I .35 \text {; control: } \\
9.4 \pm 2.0\end{array}$ & $\begin{array}{l}\text { Double-blind, } \\
\text { placebo-controlled } \\
\text { randomized clinical } \\
\text { trial } \\
\text { Intervention: } \\
25 \mathrm{mg} / 100 \mathrm{mg} \\
\text { of carbidopa and } \\
\text { levodopa, } \\
\text { increased up to } 250 \text { - } \\
600 \mathrm{mg} \text { of levodopa vs } \\
\text { placebo for the ADHD } \\
\text { group }\end{array}$ & $\begin{array}{l}\text { ADHD: DISC-IV } \\
\text { RLS: PSG }\end{array}$ & Sleep: PSG \\
\hline $\begin{array}{l}\text { Akinci et al } \\
(2015)^{84}\end{array}$ & $\begin{array}{l}28 \text { children with ADHD and I5 } \\
\text { healthy controls } \\
\text { Sex: } 29 \text { males, } 14 \text { females } \\
\text { Age (median [age range]), years: } \\
\text { ADHD: } 10 \text { (9-13); control: } 10 \\
(8-12)\end{array}$ & Case-control & $\begin{array}{l}\text { ADHD: K-SADS } \\
\text { RLS: PSG }\end{array}$ & $\begin{array}{l}\text { Sleep: PSG, PSQI } \\
\text { Sleepiness: ESS }\end{array}$ \\
\hline $\begin{array}{l}\text { Garbazza et al } \\
(20 \mid 8)^{85}\end{array}$ & $\begin{array}{l}\text { I5 ADHD adults without RLS/ } \\
\text { PLMD and I } 8 \text { healthy controls } \\
\text { Sex: } 14 \text { males, } 19 \text { females } \\
\text { Age (mean } \pm \text { SD), years: } \\
\text { ADHD: } 33.9 \pm 7.9 \text {; controls: } \\
35.8 \pm 7.5\end{array}$ & Case-control & $\begin{array}{l}\text { ADHD: DSM-IV } \\
\text { criteria } \\
\text { RLS: RLDI }\end{array}$ & Sleep: PSG \\
\hline \multicolumn{5}{|c|}{ Table I.3 Circadian rhythm sleep disorders } \\
\hline $\begin{array}{l}\text { Bijlenga et al } \\
(20 \mid 3)^{91}\end{array}$ & $\begin{array}{l}\text { I2 medication naïve adults with } \\
\text { ADHD and DSPS, } 12 \text { healthy } \\
\text { controls } \\
\text { Sex: } 12 \text { males, } 12 \text { females } \\
\text { Age (M } \pm \text { SD), years: ADHD } \\
+ \text { DSPS: } 32.5 \pm 9.9 \text {; control: } \\
32.4 \pm 10.8\end{array}$ & Case-control & $\begin{array}{l}\text { ADHD: DIVA } 2.0 \\
\text { DSPS: inability } \\
\text { to fall asleep at } \\
\text { preferred time } \\
\text { before } 23: 30 \text { in the } \\
\text { past } 6 \text { months }\end{array}$ & $\begin{array}{l}\text { Sleep: MCTQ, } \\
\text { SHQ, actigraphy } \\
\text { Circadian rhythms: } \\
\text { DLMO, CBT, } \\
\text { actigraphy }\end{array}$ \\
\hline
\end{tabular}




\begin{tabular}{|c|c|c|c|c|}
\hline \multirow[t]{2}{*}{ Sleep differences } & \multicolumn{3}{|l|}{ Daytime impairments } & \multirow[t]{2}{*}{ Conclusions } \\
\hline & Cognitive & Emotional & Physical & \\
\hline $\begin{array}{l}\text { RLS + ADHD vs RLS: no } \\
\text { difference in serum ferritin and } \\
\text { iron levels. }\end{array}$ & $\begin{array}{l}27.6 \% \text { of patients with RLS } \\
\text { have comorbid ADHD. }\end{array}$ & $\begin{array}{l}\text { RLS + ADHD } \\
\text { vs RLS: } \\
\text { prevalence } \\
\text { of OCD is } \\
7.62 \% \text { for both } \\
\text { groups. }\end{array}$ & $\mathrm{N} / \mathrm{A}$ & $\begin{array}{l}\text { ADHD is highly comorbid } \\
\text { with RLS. OCD } \\
\text { comorbidity may also be } \\
\text { higher in those with RLS. }\end{array}$ \\
\hline $\begin{array}{l}\text { ADHD vs control: higher PLMD } \\
\text { index and lower periodicity of } \\
\text { leg movements. No changes } \\
\text { with treatment in periodicity of } \\
\text { movement. }\end{array}$ & $\mathrm{N} / \mathrm{A}$ & $\mathrm{N} / \mathrm{A}$ & $\mathrm{N} / \mathrm{A}$ & $\begin{array}{l}\text { Children with ADHD had } \\
\text { more PLMD symptoms } \\
\text { than controls. } \\
\text { Treatment with levodopa } \\
\text { and carbidopa did not } \\
\text { change RLS symptoms. }\end{array}$ \\
\hline $\begin{array}{l}\text { ADHD vs control: PLMI was } \\
\text { higher. No differences in } \\
\text { subjective perception of RLS. }\end{array}$ & $\mathrm{N} / \mathrm{A}$ & $\mathrm{N} / \mathrm{A}$ & $\mathrm{N} / \mathrm{A}$ & $\begin{array}{l}\text { RLS is greater in children } \\
\text { with ADHD than in healthy } \\
\text { children. }\end{array}$ \\
\hline $\begin{array}{l}\text { ADHD vs control: longer sleep } \\
\text { latency, longer duration of } \\
\text { periodic leg movements during } \\
\text { sleep, and higher PLMS index in } \\
\text { REM sleep. }\end{array}$ & $\mathrm{N} / \mathrm{A}$ & $\mathrm{N} / \mathrm{A}$ & $\mathrm{N} / \mathrm{A}$ & $\begin{array}{l}\text { Adults with ADHD } \\
\text { but without RLS/ } \\
\text { PLMD present, greater } \\
\text { subthreshold PLMD } \\
\text { symptoms than healthy } \\
\text { controls. }\end{array}$ \\
\hline $\begin{array}{l}\text { Sleep: ADHD + DSPS vs } \\
\text { controls: midpoint of sleep } \\
\text { was 1:52 hours later, bed time } \\
\text { was 2:23 hours later, sleep } \\
\text { start time was 2:20 hours } \\
\text { later, sleep duration was I:08 } \\
\text { hours shorter on work days, } \\
\text { sleep hygiene was worse, sleep } \\
\text { efficiency was better by } 4.5 \\
\text { percentage points. Circadian } \\
\text { rhythms: ADHD + DPSP } \\
\text { vs controls: DLMO onset } \\
\text { occurred I:23 hours later; } \\
\text { 24-hour activity parameters } \\
\text { were delayed. CBT and } \\
\text { distal skin temperature were } \\
\text { lower, while proximal skin } \\
\text { temperature was higher. }\end{array}$ & $\mathrm{N} / \mathrm{A}$ & N/A & $\mathrm{N} / \mathrm{A}$ & $\begin{array}{l}\text { Circadian rhythms of } \\
\text { melatonin and activity were } \\
\text { delayed in participants with } \\
\text { ADHD and DSPS. }\end{array}$ \\
\hline
\end{tabular}

(Continued) 
Table I (Continued)

\begin{tabular}{|c|c|c|c|c|}
\hline Author and year & Sample & Study design & Diagnoses & Measures \\
\hline $\begin{array}{l}\text { Durmuș et al } \\
(2017)^{92}\end{array}$ & $\begin{array}{l}52 \text { children with ADHD, } 52 \\
\text { healthy controls } \\
\text { Sex: } 82 \text { males, } 22 \text { females } \\
\text { Age }(M \pm S D) \text {, years: ADHD: } \\
9.24 \pm 1.94 \text {; control: } 9.20 \pm 1.20\end{array}$ & Case-control & $\begin{array}{l}\text { ADHD: K-SADS-PL } \\
\text { Sleep: None }\end{array}$ & $\begin{array}{l}\text { Sleep: CHSQ } \\
\text { Chronotype: } \\
\text { CCTQ } \\
\text { Attention: CPRS, } \\
\text { CTRS, DSM-IV } \\
\text { symptom checklist. } \\
\text { Intelligence: } \\
\text { WISC-R }\end{array}$ \\
\hline $\begin{array}{l}\text { Snitselaar et al } \\
(20 \mid 3)^{90}\end{array}$ & $\begin{array}{l}10 \text { adults with ADHD } \\
\text { Sex: } 8 \text { males, } 2 \text { females } \\
\text { Age (mean } \pm \text { SD), years: } \\
33.2 \pm 7.6\end{array}$ & $\begin{array}{l}\text { Open-label trial } \\
\text { Treatment: MPH }\end{array}$ & $\begin{array}{l}\text { ADHD: DSM-IV } \\
\text { criteria } \\
\text { Sleep: None }\end{array}$ & $\begin{array}{l}\text { Chronotype: } \\
\text { MCTQ, DLMO } \\
\text { Attention: ADHD- } \\
\text { RS }\end{array}$ \\
\hline $\begin{array}{l}\text { Büber et al } \\
(2016)^{88}\end{array}$ & $\begin{array}{l}27 \text { children and adolescents } \\
\text { with ADHD and } 28 \text { healthy } \\
\text { controls. } \\
\text { Sex: } 44 \text { males, II females } \\
\text { Age (mean } \pm \text { SD), years: } \\
\text { ADHD: } 9.37 \pm 2.69 \text {; control: } \\
|0.50 \pm 2.7|\end{array}$ & Case-control & $\begin{array}{l}\text { ADHD: K-SADS-PL } \\
\text { Sleep: None }\end{array}$ & $\begin{array}{l}\text { Chronotype: } \\
\text { urinary 6-OH MS } \\
\text { Attention: CPRS }\end{array}$ \\
\hline Bron et al $(2016)^{40}$ & $\begin{array}{l}2,090 \text { adults } \\
\text { Sex: } 696 \text { males, I,394 females } \\
\text { Age (mean } \pm \text { SD), years: lifetime } \\
\text { depression or anxiety + ADHD: } \\
47.6 \pm 11.3 \text {; lifetime depression } \\
\text { or anxiety: } 44.6 \pm 12.8 \text {; controls: } \\
44.0 \pm 14.6\end{array}$ & Cross-sectional & $\begin{array}{l}\text { ADHD: CAARS } \\
\text { DSPS: MCTQ }\end{array}$ & $\begin{array}{l}\text { Chronotype: } \\
\text { MCTQ } \\
\text { Attention: CAARS } \\
\text { Socioemotional: } \\
\text { CIDI } \\
\text { Physical: BMI }\end{array}$ \\
\hline Bumb et al $(2016)^{95}$ & $\begin{array}{l}74 \text { adults with ADHD, } 86 \\
\text { healthy controls. } \\
\text { Sex: } 82 \text { males, } 78 \text { females } \\
\text { Age (mean } \pm \text { SD), years: } \\
\text { ADHD: } 32.7 \pm 8.1 \text {; control: } \\
23.1 \pm 2.6\end{array}$ & Case-control & $\begin{array}{l}\text { ADHD: ADHD-DC } \\
\text { Sleep: none }\end{array}$ & $\begin{array}{l}\text { Chronotype: MEQ } \\
\text { Attention: ADHD- } \\
\text { DC, WURS-K } \\
\text { Intelligence: } \\
\text { MWT-B }\end{array}$ \\
\hline $\begin{array}{l}\text { Molina-Carballo } \\
\text { et al }(2013)^{89}\end{array}$ & $\begin{array}{l}\text { I } 36 \text { children with ADHD group, } \\
42 \text { healthy controls. } \\
\text { Sex: I } 36 \text { males, } 42 \text { females } \\
\text { Age (mean } \pm \text { SD), years: } \\
9.70 \pm 2.55\end{array}$ & $\begin{array}{l}\text { Quasiexperimental } \\
\text { open-label trial } \\
\text { Intervention: extended } \\
\text { release MPH }\end{array}$ & $\begin{array}{l}\text { ADHD: DSM-IV or } \\
\text { ICD-9 criteria } \\
\text { Sleep: none }\end{array}$ & $\begin{array}{l}\text { Sleep: sleep diary } \\
\text { Attention: EDAH } \\
\text { Chronotype: } \\
\text { urinary 6-OH MS } \\
\text { Serum melatonin } \\
\text { Socioemotional: } \\
\text { CDI } \\
\text { Serum serotonin }\end{array}$ \\
\hline
\end{tabular}




\begin{tabular}{|c|c|c|c|c|}
\hline \multirow[t]{2}{*}{ Sleep differences } & \multicolumn{3}{|l|}{ Daytime impairments } & \multirow[t]{2}{*}{ Conclusions } \\
\hline & Cognitive & Emotional & Physical & \\
\hline $\begin{array}{l}\text { ADHD vs control: more } \\
\text { eveningness and worse sleep } \\
\text { hygiene. In the ADHD group, } \\
\text { eveningness was associated } \\
\text { with the following subscales in } \\
\text { the CSHQ: resistance to sleep, } \\
\text { respiratory problems, daytime } \\
\text { sleepiness, and total score. In } \\
\text { the control group, eveningness } \\
\text { was only associated with } \\
\text { daytime sleepiness. }\end{array}$ & $\begin{array}{l}\text { In the ADHD group, somatic } \\
\text { symptoms in the CPRS were } \\
\text { associated with eveningness. } \\
\text { ADHD vs control: lower } \\
\text { WISC-R scores. }\end{array}$ & $\mathrm{N} / \mathrm{A}$ & $\mathrm{N} / \mathrm{A}$ & $\begin{array}{l}\text { Children with ADHD had } \\
\text { greater eveningness, which } \\
\text { was associated with worse } \\
\text { sleep hygiene. Eveningness } \\
\text { was associated with worse } \\
\text { daytime somatic symptoms } \\
\text { of ADHD. }\end{array}$ \\
\hline $\begin{array}{l}\text { Circadian rhythms: MPH } \\
\text { increased melatonin levels but } \\
\text { did not change the timing of } \\
\text { DLMO. }\end{array}$ & $\begin{array}{l}\text { Morningness was associated } \\
\text { with greater reduction } \\
\text { of symptoms after MPH } \\
\text { treatment. }\end{array}$ & $\mathrm{N} / \mathrm{A}$ & & $\begin{array}{l}\text { Patients with a morning } \\
\text { type may have more } \\
\text { improvement in ADHD } \\
\text { symptoms than those with } \\
\text { the evening type. }\end{array}$ \\
\hline $\begin{array}{l}\text { Circadian rhythms: ADHD } \\
\text { vs control: higher levels of } \\
\text { daytime, nighttime, and 24-hour } \\
\text { melatonin. }\end{array}$ & $\begin{array}{l}\text { Levels of 6-OH MS were not } \\
\text { associated with symptom } \\
\text { severity. }\end{array}$ & N/A & $\mathrm{N} / \mathrm{A}$ & $\begin{array}{l}\text { While melatonin levels } \\
\text { are associated with the } \\
\text { presence of ADHD, no } \\
\text { evidence suggests that } \\
\text { it was associated with } \\
\text { daytime symptom severity } \\
\text { or subtype. }\end{array}$ \\
\hline $\begin{array}{l}\text { Lifetime depression or anxiety } \\
+ \text { ADHD vs controls or lifetime } \\
\text { depression or anxiety: shorter } \\
\text { sleep duration (OR=2.8). } \\
\text { Lifetime depression or anxiety } \\
+ \text { ADHD vs lifetime depression } \\
\text { or anxiety: higher prevalence } \\
\text { of late chronotype (OR }=2.6) \\
\text { and DSPS (OR }=2.4) \text {. For } \\
\text { every SD increase of inattentive } \\
\text { symptoms or ADHD symptoms } \\
\text { index, OR for DSPS and } \\
\text { extreme evening chronotype } \\
\text { increased by I.3. }\end{array}$ & $\mathrm{N} / \mathrm{A}$ & $\mathrm{N} / \mathrm{A}$ & $\begin{array}{l}\text { No } \\
\text { differences } \\
\text { in BMI } \\
\text { between } \\
\text { groups. }\end{array}$ & $\begin{array}{l}\text { ADHD symptoms, } \\
\text { specifically inattentive } \\
\text { symptoms, are associated } \\
\text { with extreme evening } \\
\text { chronotype in the presence } \\
\text { of lifetime depression and } \\
\text { anxiety. }\end{array}$ \\
\hline $\mathrm{N} / \mathrm{A}$ & $\begin{array}{l}\text { ADHD vs controls: greater } \\
\text { eveningness. Eveningness } \\
\text { was associated with greater } \\
\text { ADHD symptoms. }\end{array}$ & $\mathrm{N} / \mathrm{A}$ & $\mathrm{N} / \mathrm{A}$ & $\begin{array}{l}\text { Eveningness is associated } \\
\text { with more symptoms of } \\
\text { ADHD. }\end{array}$ \\
\hline $\begin{array}{l}\text { Circadian rhythms: MPH } \\
\text { treatment reduced urinary } \\
6-\mathrm{OH} \text { MS excretion and } \\
\text { lowered morning melatonin in } \\
\text { ADHD patients. }\end{array}$ & $\begin{array}{l}\text { ADHD symptoms improve } \\
\text { after MPH treatment. }\end{array}$ & $\begin{array}{l}\text { CDI improves } \\
\text { after MPH } \\
\text { treatment. } \\
\text { Serotonin } \\
\text { was lower in } \\
\text { ADHD patients } \\
\text { in the morning } \\
\text { compared } \\
\text { with controls; } \\
\text { serotonin was } \\
\text { not affected by } \\
\text { MPH. }\end{array}$ & $\mathrm{N} / \mathrm{A}$ & $\begin{array}{l}\text { Stimulant treatment of } \\
\text { ADHD reduces melatonin } \\
\text { levels, which may have } \\
\text { an impact on DSPS or } \\
\text { chronotype. }\end{array}$ \\
\hline
\end{tabular}

(Continued) 
Table I (Continued)

\begin{tabular}{|c|c|c|c|c|}
\hline Author and year & Sample & Study design & Diagnoses & Measures \\
\hline \multicolumn{5}{|l|}{ Table I.4 Insomnia } \\
\hline $\begin{array}{l}\text { Vélez-Galarraga et } \\
\text { al }(2016)^{99}\end{array}$ & $\begin{array}{l}\text { I } 26 \text { children with ADHD, I,036 } \\
\text { controls } \\
\text { Sex: } 504 \text { males, } 658 \text { females } \\
\text { Age (mean } \pm \text { SD), years: } \\
\text { ADHD: II.25 } \pm 3.25 \text {; controls: } \\
\text { I0.83 } \pm 3.75\end{array}$ & Case-control & $\begin{array}{l}\text { ADHD: K-SADS- } \\
\text { PL, DSM-IV criteria } \\
\text { Insomnia: PSG }\end{array}$ & $\begin{array}{l}\text { Sleep: PSG } \\
\text { Attention: ADHD- } \\
\text { RS, CPT }\end{array}$ \\
\hline $\begin{array}{l}\text { Yoon et al } \\
(2013)^{101}\end{array}$ & $\begin{array}{l}126 \text { adults with ADHD } \\
\text { Sex: } 93 \text { males, } 33 \text { females } \\
\text { Age (mean } \pm S D \text { ), years: } \\
38.1 \pm 10.4\end{array}$ & Cross-sectional & $\begin{array}{l}\text { ADHD: CAARS- } \\
\text { S:L, ASRS, SCID-I, } \\
\text { TCI } \\
\text { Insomnia: PSQI }\end{array}$ & $\begin{array}{l}\text { Sleep: PSQI } \\
\text { Sleepiness: ESS, FSS } \\
\text { Attention: CAARS- } \\
\text { S:L, ASRS }\end{array}$ \\
\hline $\begin{array}{l}\text { Moreau et al } \\
(2014)^{97}\end{array}$ & $\begin{array}{l}4 \mathrm{I} \text { children with ADHD, } 4 \mathrm{I} \\
\text { controls } \\
\text { Sex: } 24 \text { males, I } 7 \text { females } \\
\text { Age (mean } \pm \text { SD), years: } \\
\text { ADHD: } 9.74 \pm \mathrm{I} .69 \text {; controls: } \\
9.57 \pm \mathrm{I} .62\end{array}$ & Case-control & $\begin{array}{l}\text { ADHD: CPRS-R, } \\
\text { CTRS-R, K-SADS- } \\
\text { PL } \\
\text { Insomnia: ISI-C }\end{array}$ & $\begin{array}{l}\text { Sleep: Actigraphy, } \\
\text { CSHQ, ISI-C } \\
\text { Attention: CPRS, } \\
\text { CTRS, CBCL } \\
\text { Socioemotional: } \\
\text { CBCL }\end{array}$ \\
\hline $\begin{array}{l}\text { Corkum et al } \\
(2016)^{98}\end{array}$ & $\begin{array}{l}22 \text { children with ADHD and } \\
39 \text { typically developing with } \\
\text { behavioral insomnia } \\
\text { Sex: } 28 \text { males, } 33 \text { females } \\
\text { Age (mean } \pm S D \text { ), years: } \\
9.11 \pm 1.96\end{array}$ & $\begin{array}{l}\text { Randomized } \\
\text { controlled trial } \\
\text { Intervention: "Better } \\
\text { Nights/Better Days" } \\
\text { distance sleep } \\
\text { intervention or } \\
\text { waitlist control }\end{array}$ & $\begin{array}{l}\text { ADHD: K-SADS-PL } \\
\text { Insomnia: DSM-IV } \\
\text { criteria }\end{array}$ & $\begin{array}{l}\text { Sleep: CHSQ } \\
\text { Actigraphy } \\
\text { Sleep evaluation } \\
\text { questionnaire } \\
\text { Attention: CBCL } \\
\text { Socioemotional: } \\
\text { CBCL }\end{array}$ \\
\hline $\begin{array}{l}\text { Brevik et al } \\
(2017)^{47}\end{array}$ & $\begin{array}{l}268 \text { adults with ADHD, } 202 \\
\text { controls } \\
\text { Sex: I } 84 \text { males, } 286 \text { females } \\
\text { Age (mean } \pm \text { SD), years: } \\
\text { ADHD: } 38.1 \pm \text { I I.4; controls: } \\
36.5 \pm 8.0\end{array}$ & Case-control & $\begin{array}{l}\text { ADHD: ASRS } \\
\text { Insomnia: BIS }\end{array}$ & $\begin{array}{l}\text { Sleep: BIS } \\
\text { Attention: ASRS }\end{array}$ \\
\hline
\end{tabular}




\begin{tabular}{|c|c|c|c|c|}
\hline \multirow[t]{2}{*}{ Sleep differences } & \multicolumn{3}{|l|}{ Daytime impairments } & \multirow[t]{2}{*}{ Conclusions } \\
\hline & Cognitive & Emotional & Physical & \\
\hline $\begin{array}{l}\text { ADHD vs controls: greater } \\
\text { bedtime resistance, difficulty } \\
\text { falling asleep, and sleep onset } \\
\text { latencies longer than } 30 \text { minutes } \\
\text { than controls. No differences in } \\
\text { insomnia prevalence. }\end{array}$ & $\begin{array}{l}\text { In children with ADHD: } \\
\text { ADHD-RS } \geq 24 \text { vs ADHD } \\
<24: \text { higher prevalence of } \\
\text { insomnia. Higher omission } \\
\text { errors on the CPT were } \\
\text { associated with shorter sleep } \\
\text { duration. }\end{array}$ & $\mathrm{N} / \mathrm{A}$ & $\mathrm{N} / \mathrm{A}$ & $\begin{array}{l}\text { In children with ADHD, } \\
\text { more symptoms of } \\
\text { ADHD were associated } \\
\text { with a higher prevalence } \\
\text { of insomnia, and more } \\
\text { inattentive errors on the } \\
\text { CPT were associated } \\
\text { with shorter sleep } \\
\text { duration. }\end{array}$ \\
\hline $\begin{array}{l}\text { Initial insomnia was the most } \\
\text { common subjective complaint } \\
\text { in adults with ADHD. ADHD-I } \\
\text { vs ADHD-C: no differences } \\
\text { in proportion of insomnia or } \\
\text { sleepiness; greater PSQI scores } \\
\text { and fatigue. }\end{array}$ & $N / A$ & $\mathrm{~N} / \mathrm{A}$ & $\mathrm{N} / \mathrm{A}$ & $\begin{array}{l}\text { ADHD-I was associated } \\
\text { with greater daytime } \\
\text { fatigue and greater sleep } \\
\text { disturbance. }\end{array}$ \\
\hline $\begin{array}{l}\text { Children with ADHD vs } \\
\text { controls: increased sleep } \\
\text { onset latency and night } \\
\text { awakenings, higher insomnia } \\
\text { severity, shorter actigraphic } \\
\text { sleep time, longer sleep onset } \\
\text { latency, lower sleep efficiency, } \\
\text { and greater variability of } \\
\text { sleep onset latency. Having a } \\
\text { comorbid psychiatric disorder } \\
\text { (in addition to ADHD) } \\
\text { worsened these measures. } \\
\text { Stimulant medication use } \\
\text { was not associated with any } \\
\text { differences in sleep. }\end{array}$ & $\mathrm{N} / \mathrm{A}$ & $\mathrm{N} / \mathrm{A}$ & $\mathrm{N} / \mathrm{A}$ & $\begin{array}{l}\text { Presence of ADHD is } \\
\text { associated with increased } \\
\text { sleep problems and } \\
\text { insomnia severity. }\end{array}$ \\
\hline $\begin{array}{l}\text { Sleep intervention improved } \\
\text { objective and subjective sleep } \\
\text { onset latency, subjective sleep } \\
\text { duration, bedtime resistance, } \\
\text { and total sleep disturbance } \\
\text { (vs no changes in waitlist } \\
\text { control). This improvement } \\
\text { was maintained at 6-month } \\
\text { follow-up. }\end{array}$ & $\begin{array}{l}\text { Sleep intervention improved } \\
\text { CBCL scores, which } \\
\text { includes attention problems. } \\
\text { Improvement increased at } \\
\text { 6-month follow-up. }\end{array}$ & $\begin{array}{l}\text { Sleep } \\
\text { intervention } \\
\text { improved } \\
\mathrm{CBCL} \\
\text { internalizing } \\
\text { and } \\
\text { externalizing } \\
\text { scores. This } \\
\text { improvement } \\
\text { increased } \\
\text { at 6-month } \\
\text { follow-up. }\end{array}$ & $\mathrm{N} / \mathrm{A}$ & $\begin{array}{l}\text { Better nights/better days } \\
\text { was effective in improving } \\
\text { sleep and daytime } \\
\text { functioning in children with } \\
\text { insomnia that are either } \\
\text { typically developing or with } \\
\text { ADHD. }\end{array}$ \\
\hline $\begin{array}{l}\text { ADHD vs controls: } 67 \% \text { vs } 29 \% \\
\text { insomnia prevalence. } \\
\text { ADHD-C vs ADHD-l: higher } \\
\text { prevalence of insomnia. } \\
\text { Stimulant treatment vs no } \\
\text { treatment: lower insomnia } \\
\text { severity. }\end{array}$ & $\begin{array}{l}\text { Insomnia severity was } \\
\text { strongly correlated with } \\
\text { inattentive symptom severity. }\end{array}$ & $\mathrm{N} / \mathrm{A}$ & $\mathrm{N} / \mathrm{A}$ & $\begin{array}{l}\text { Insomnia is more prevalent } \\
\text { in adults with ADHD, } \\
\text { specifically in ADHD-C. } \\
\text { Inattentive symptoms are } \\
\text { correlated with insomnia } \\
\text { severity. }\end{array}$ \\
\hline
\end{tabular}

(Continued) 
Table I (Continued)

\begin{tabular}{|c|c|c|c|c|}
\hline Author and year & Sample & Study design & Diagnoses & Measures \\
\hline \multicolumn{5}{|l|}{ Table I.5 Narcolepsy } \\
\hline Ito et al $(2018)^{57}$ & $\begin{array}{l}77 \text { adolescent and adult } \\
\text { outpatients with narcolepsy } \\
\text { type } 2 \text { or hypersomnia. } \\
\text { Sex: } 48 \text { males, } 29 \text { females } \\
\text { Age }(M \pm S D) \text {, years: narcolepsy } \\
\text { type } 2: 25.7 \pm 5.8 \text {; narcolepsy } \\
\text { type } 2+\text { ADHD: } 23.0 \pm 7.0\end{array}$ & Cross-sectional & $\begin{array}{l}\text { ADHD: DSM-IV } \\
\text { criteria } \\
\text { Narcolepsy type } \\
\text { 2: self-reported } \\
\text { symptoms and } \\
\text { MSLT }\end{array}$ & $\begin{array}{l}\text { Sleep: PSG } \\
\text { Sleepiness: MSLT }\end{array}$ \\
\hline $\begin{array}{l}\text { Lecendreux et al } \\
(2015)^{106}\end{array}$ & $\begin{array}{l}\text { I } 88 \text { children with narcolepsy. } \\
67 \text { healthy controls. } \\
\text { Sex: } 85 \text { males, } 90 \text { females } \\
\text { Age (median [range]), years: } \\
\text { narcolepsy type I: } 14.0 \\
(6.6-17.8) \text {; narcolepsy type } 2 \text { : } \\
\text { I0.3 (5.9-17.4); controls: I4.8 } \\
(7.0-17.9)\end{array}$ & Case-control & $\begin{array}{l}\text { ADHD: ADHD-RS } \\
\text { Narcolepsy: ICSD-2 } \\
\text { criteria, PSG, MSLT, } \\
\text { CSF hypocretin-I } \\
\text { levels }\end{array}$ & $\begin{array}{l}\text { Sleep: ISI } \\
\text { Sleepiness: PDSS } \\
\text { Attention: ADHD- } \\
\text { RS } \\
\text { Socioemotional: } \\
\text { CDI }\end{array}$ \\
\hline $\begin{array}{l}\text { Filardi et al } \\
(2017)^{108}\end{array}$ & $\begin{array}{l}21 \text { adults with narcolepsy type } \\
\text { I, I5 adults with narcolepsy } \\
\text { type } 2 \text {, and } 22 \text { healthy controls } \\
\text { Sex: } 26 \text { males, } 32 \text { females } \\
\text { Age (M } \pm S D) \text {, years: narcolepsy } \\
\text { type I: } 36 \pm 12 \text {; narcolepsy type } \\
2: 36 \pm 13 \text {; controls: } 35 \pm 12\end{array}$ & Cross-sectional & $\begin{array}{l}\text { ADHD: ASRS } \\
\text { Narcolepsy: ICSD-3 } \\
\text { criteria }\end{array}$ & $\begin{array}{l}\text { Sleep: narcolepsy: } \\
\text { PSG; controls: } \\
\text { actigraphy. } \\
\text { Sleepiness: ESS } \\
\text { MSLT } \\
\text { Attention } \\
\text { ASRS; ANT } \\
\text { Socioemotional: } \\
\text { BDI; STAI; OCIr } \\
\text { Physical: BMI. }\end{array}$ \\
\hline $\begin{array}{l}\text { Rocca et al } \\
(2016)^{104}\end{array}$ & $\begin{array}{l}29 \text { children with narcolepsy } \\
\text { type I, } 39 \text { controls. } \\
\text { Sex: } 36 \text { males, } 32 \text { females } \\
\text { Age (M } \pm S D) \text {, years: narcolepsy } \\
\text { type I: I I.52 } \pm 2.89 \text {; control: } \\
\text { I I. } 2 \text { I } \pm 3.44\end{array}$ & Case-control & $\begin{array}{l}\text { ADHD: CBCL } \\
\text { Narcolepsy: } \\
\text { previous diagnosis } \\
\text { by psychiatrist } \\
\text { (criteria not } \\
\text { specified) }\end{array}$ & $\begin{array}{l}\text { Sleep: PSG } \\
\text { Attention: CBCL } \\
\text { Quality of life: } \\
\text { PedsQL }\end{array}$ \\
\hline $\begin{array}{l}\text { Zamarian et al } \\
(2015)^{107}\end{array}$ & $\begin{array}{l}5 \text { I narcolepsy. } 35 \text { healthy } \\
\text { controls. } \\
\text { Sex: } 46 \text { males, } 40 \text { females } \\
\text { Age }(M \pm S D) \text {, years: narcolepsy } \\
\text { type: } 38.94 \pm 14.15 \text {. Control: } \\
39.86 \pm 13.96\end{array}$ & Cross-sectional & $\begin{array}{l}\text { ADHD: None } \\
\text { Narcolepsy: ICSD-2 }\end{array}$ & $\begin{array}{l}\text { Sleepiness: SSS } \\
\text { Attention: } \\
\text { Questionnaire on } \\
\text { perceived deficits of } \\
\text { attention, digit span, } \\
\text { backward digit span, } \\
\text { D2 attention test, } \\
\text { RWT, IED, SOC, } \\
\text { go/no go, auditory } \\
\text { verbal learning test } \\
\text { Socioemotional: } \\
\text { HADS }\end{array}$ \\
\hline
\end{tabular}




\begin{tabular}{|c|c|c|c|c|}
\hline \multirow[t]{2}{*}{ Sleep differences } & \multicolumn{3}{|l|}{ Daytime impairments } & \multirow[t]{2}{*}{ Conclusions } \\
\hline & Cognitive & Emotional & Physical & \\
\hline $\begin{array}{l}\text { Sleep: shorter stage NI, longer } \\
\text { stage N3 in narcolepsy + } \\
\text { ADHD compared with those } \\
\text { with only narcolepsy } \\
\text { Sleepiness: narcolepsy + ADHD } \\
\text { shorter REM sleep latencies } \\
\text { on MSLT than those with only } \\
\text { narcolepsy. }\end{array}$ & $\mathrm{N} / \mathrm{A}$ & N/A & $\mathrm{N} / \mathrm{A}$ & $\begin{array}{l}\text { Narcolepsy type } 2+ \\
\text { ADHD vs narcolepsy: } \\
\text { greater daytime sleepiness. }\end{array}$ \\
\hline $\begin{array}{l}\text { In children with narcolepsy, } \\
\text { ADHD symptoms were } \\
\text { associated with longer sleep } \\
\text { onset latencies. }\end{array}$ & $\begin{array}{l}\text { Narcolepsy vs controls: more } \\
\text { ADHD symptoms. } \\
\text { Insomnia and fatigue were } \\
\text { associated with increased } \\
\text { inattention. }\end{array}$ & $\begin{array}{l}\text { Narcolepsy } \\
\text { + ADHD vs } \\
\text { narcolepsy: } \\
\text { significantly } \\
\text { more } \\
\text { depressive } \\
\text { symptoms and } \\
\text { lower quality } \\
\text { of life. }\end{array}$ & $\mathrm{N} / \mathrm{A}$ & $\begin{array}{l}\text { Narcolepsy vs controls: } \\
\text { more symptoms of ADHD. } \\
\text { More ADHD symptoms } \\
\text { are associated with more } \\
\text { depressive symptoms. }\end{array}$ \\
\hline $\begin{array}{l}\text { In narcolepsy type I, impaired } \\
\text { attention was associated with } \\
\text { greater sleepiness. }\end{array}$ & $\begin{array}{l}\text { Narcolepsy vs controls: } \\
\text { more inattentive and slower } \\
\text { reaction times. } \\
\text { Narcolepsy type I vs } \\
\text { narcolepsy type } 2 \text { and } \\
\text { controls: more hyperactive } \\
\text { and worse alerting scores. }\end{array}$ & $\begin{array}{l}\text { In narcolepsy } \\
\text { type I, higher } \\
\text { ADHD } \\
\text { symptoms } \\
\text { were } \\
\text { associated } \\
\text { with higher } \\
\text { depression } \\
\text { scores. }\end{array}$ & $\begin{array}{l}\text { Narcolepsy } \\
\text { type I vs } \\
\text { narcolepsy } \\
\text { type } 2 \text { and } \\
\text { controls: } \\
\text { higher BMI }\end{array}$ & $\begin{array}{l}\text { Narcolepsy vs controls: } \\
\text { greater ADHD symptoms. } \\
\text { ADHD symptoms in } \\
\text { narcolepsy were associated } \\
\text { with more depressive } \\
\text { symptoms. }\end{array}$ \\
\hline $\begin{array}{l}\text { Total sleep time of } 489 \pm 72 \\
\text { minutes in the narcolepsy } \\
\text { group. Total sleep time was not } \\
\text { reported in the control group. }\end{array}$ & $\begin{array}{l}\text { Narcolepsy type I was } \\
\text { associated with higher } \\
\text { attention problems and } \\
\text { ADHD scores. Treatment } \\
\text { with sodium oxybate, but } \\
\text { not with modafinil, was } \\
\text { associated with improved } \\
\text { attention and ADHD scores. }\end{array}$ & $\begin{array}{l}\text { In participants } \\
\text { with narcolepsy } \\
\text { type I, ADHD } \\
\text { symptoms } \\
\text { were } \\
\text { associated with } \\
\text { worse school } \\
\text { functioning } \\
\text { and higher } \\
\text { psychosocial } \\
\text { health. }\end{array}$ & $\begin{array}{l}\text { In the } \\
\text { narcolepsy } \\
\text { type I } \\
\text { group, } \\
\text { there } \\
\text { were no } \\
\text { significant } \\
\text { associations } \\
\text { between } \\
\text { ADHD } \\
\text { symptoms } \\
\text { and physical } \\
\text { health. }\end{array}$ & $\begin{array}{l}\text { Narcolepsy vs controls: } \\
\text { more ADHD symptoms. } \\
\text { ADHD symptoms in } \\
\text { narcolepsy are associated } \\
\text { with worse school and } \\
\text { psychosocial health. } \\
\text { Sodium oxybate treatment } \\
\text { of narcolepsy is associated } \\
\text { with reduced daytime } \\
\text { impairment. }\end{array}$ \\
\hline $\begin{array}{l}\text { Sleepiness increased with } \\
\text { testing in the narcolepsy group } \\
\text { but not in the control group. }\end{array}$ & $\begin{array}{l}\text { Narcolepsy vs control: } \\
\text { greater subjective deficits } \\
\text { of attention, worse } \\
\text { selective attention, reduced } \\
\text { verbal fluency, and fewer } \\
\text { participants reached the go } \\
\text { ceiling in the go/no go task. } \\
\text { Subjective deficit of attention } \\
\text { was associated with } \\
\text { sleepiness and depression. }\end{array}$ & $\begin{array}{l}\text { Narcolepsy } \\
\text { vs control: } \\
\text { greater } \\
\text { depression. }\end{array}$ & $\mathrm{N} / \mathrm{A}$ & $\begin{array}{l}\text { Narcolepsy was associated } \\
\text { with worse deficits of } \\
\text { attention. }\end{array}$ \\
\hline
\end{tabular}

(Continued) 
Table I (Continued)

\begin{tabular}{|c|c|c|c|c|}
\hline Author and year & Sample & Study design & Diagnoses & Measures \\
\hline \multicolumn{5}{|c|}{ Table I.6 Various sleep disorders } \\
\hline $\begin{array}{l}\text { Miano et al } \\
(20 \mid 6)^{81}\end{array}$ & $\begin{array}{l}\text { I5 children with ADHD } \\
\text { Sex: } 13 \text { males, } 2 \text { females } \\
\text { Age (mean } \pm \text { SD), years: } \\
10.6 \pm 2.2\end{array}$ & Cross-sectional & $\begin{array}{l}\text { ADHD: K-SADS-PL } \\
\text { ADHD-RS } \\
\text { Narcolepsy: ICSD-3 } \\
\text { MSLT } \\
\text { PLMD: PSG }\end{array}$ & $\begin{array}{l}\text { Sleep: actigraphy } \\
\text { CSHQ } \\
\text { Video PSG } \\
\text { Sleepiness: MSLT } \\
\text { PDSS }\end{array}$ \\
\hline Vogel et al $(2017)^{79}$ & $\begin{array}{l}942 \text { adults } \\
\text { Sex: } 256 \text { males, } 686 \text { females } \\
\text { Age (mean } \pm \text { SD), years: } \\
48.5 \pm 14.2\end{array}$ & Cross-sectional & $\begin{array}{l}\text { ADHD: ASRS } \\
\text { Sleep disorders: } \\
\text { DSISD }\end{array}$ & $\begin{array}{l}\text { Sleep: DSISD, } \\
\text { SNS, ISI, Berlin } \\
\text { questionnaire for } \\
\text { OSA } \\
\text { Chronotype: } \\
\text { MCTQ } \\
\text { Attention: ASRS }\end{array}$ \\
\hline $\begin{array}{l}\text { Hysing et al } \\
(2016)^{94}\end{array}$ & $\begin{array}{l}9846 \text { adolescents } \\
\text { Sex: } 4,594 \text { males, 5,252 females } \\
\text { Age (mean), years: } 17 . \text { SD not } \\
\text { reported }\end{array}$ & Cross-sectional & $\begin{array}{l}\text { ADHD: ASRS } \\
\text { DSPS: ICSD-R } \\
\text { Insomnia:Lichstein's } \\
\text { criteria (2003) }\end{array}$ & $\begin{array}{l}\text { Sleep: self-report } \\
\text { ADHD: ASRS } \\
\text { Socioemotional: } \\
\text { SMFQ }\end{array}$ \\
\hline $\begin{array}{l}\text { Grünwald and } \\
\text { Schlarb }(2017)^{100}\end{array}$ & $\begin{array}{l}72 \text { children with ADHD or } \\
\text { subthreshold ADHD } \\
\text { Sex: } 57 \text { males, I } 15 \text { females } \\
\text { Age (mean } \pm \text { SD), years: } \\
8.61 \pm 1.65\end{array}$ & Cross-sectional & $\begin{array}{l}\text { ADHD: DSM-5 } \\
\text { criteria } \\
\text { Sleep: CSHQ } \\
\text { Socioemotional: } \\
\text { KINDL } \\
\text { Physical: KINDL }\end{array}$ & $\begin{array}{l}\text { Sleep: CSHQ } \\
\text { Attention: FBB-HKS }\end{array}$ \\
\hline $\begin{array}{l}\text { Bjorvatn et al } \\
(20 \mid 7)^{77}\end{array}$ & $\begin{array}{l}268 \text { adults with ADHD, } 202 \\
\text { controls } \\
\text { Sex: I83 males, } 287 \text { females } \\
\text { Age (mean), years: ADHD: } 38 . I \text {; } \\
\text { controls: } 36.5 . \text { SD not reported. }\end{array}$ & Cross-sectional & $\begin{array}{l}\text { ADHD: DSM-IV } \\
\text { criteria } \\
\text { Sleep: GSAQ }\end{array}$ & Sleep: GSAQ \\
\hline
\end{tabular}




\begin{tabular}{|c|c|c|c|c|}
\hline \multirow[t]{2}{*}{ Sleep differences } & \multicolumn{3}{|l|}{ Daytime impairments } & \multirow[t]{2}{*}{ Conclusions } \\
\hline & Cognitive & Emotional & Physical & \\
\hline $\begin{array}{l}26.6 \% \text { of children with ADHD } \\
\text { had suspected narcolepsy } \\
40 \% \text { of children with ADHD } \\
\text { had PLMD }\end{array}$ & $\mathrm{N} / \mathrm{A}$ & $\mathrm{N} / \mathrm{A}$ & $\mathrm{N} / \mathrm{A}$ & $\begin{array}{l}\text { Children with ADHD may } \\
\text { have a high prevalence of } \\
\text { narcolepsy and PLMD. }\end{array}$ \\
\hline $\begin{array}{l}\text { CRSD: the OR reporting an } \\
\text { extreme evening type was I.27 } \\
\text { and I.42 for overall ADHD } \\
\text { and inattentive symptoms, } \\
\text { respectively, for each increase } \\
\text { in ADHD symptom severity. } \\
\text { Insomnia: odds of having } \\
\text { insomnia were significantly } \\
\text { higher if ADHD was present. } \\
\text { The OR of presenting insomnia } \\
\text { was I.67 if there were } \\
\text { hyperactivity symptoms with an } \\
\text { OR of I.67. Severe symptoms vs } \\
\text { none or mild: OR of I.54, } 2.42 \text {, } \\
\text { and I.55 for initial, middle, and } \\
\text { terminal insomnia, respectively. }\end{array}$ & $\begin{array}{l}\text { ADHD symptom severity } \\
\text { classified as none, medium, } \\
\text { and severe. } \\
\text { Narcolepsy: ADHD symptom } \\
\text { severity was not associated } \\
\text { with narcolepsy. } \\
\text { PLMD: PLMD was associated } \\
\text { with severe overall ADHD } \\
\text { and hyperactive symptoms. } \\
\text { OSA vs non-OSA: OR of } \\
\text { I.22 for ADHD symptoms } \\
\text { overall, I.I7 for inattention } \\
\text { symptoms, I.39 for } \\
\text { hyperactivity symptoms, } \\
\text { and I.50 for severe overall } \\
\text { ADHD symptoms. }\end{array}$ & $\mathrm{N} / \mathrm{A}$ & $\mathrm{N} / \mathrm{A}$ & $\begin{array}{l}\text { ADHD symptoms } \\
\text { are associated with } \\
\text { PLMD, extreme evening } \\
\text { chronotype, and insomnia. } \\
\text { Inattentive symptoms } \\
\text { were associated with } \\
\text { OSA and extreme evening } \\
\text { chronotype. } \\
\text { Hyperactivity symptoms } \\
\text { were associated with } \\
\text { PLMD and insomnia. }\end{array}$ \\
\hline $\begin{array}{l}\text { Severe ADHD symptoms vs } \\
\text { mild ADHD symptoms: } 7.6 \% \\
\text { vs } 2.8 \% \text { prevalence of DSPS. } \\
33.0 \% \text { vs } 11.4 \% \text { prevalence of } \\
\text { insomnia. They also had later } \\
\text { bed times, wake up times, } \\
\text { shorter sleep duration, and } \\
\text { lower sleep efficiency on both } \\
\text { weekdays and weekends. }\end{array}$ & $\begin{array}{l}\text { Shorter sleep was associated } \\
\text { with worse ASRS scores. } \\
\text { This effect was stronger for } \\
\text { inattentive symptoms than } \\
\text { hyperactive symptoms. }\end{array}$ & $\mathrm{N} / \mathrm{A}$ & $\mathrm{N} / \mathrm{A}$ & $\begin{array}{l}\text { ADHD symptoms are } \\
\text { associated with greater } \\
\text { prevalence of DSPS and } \\
\text { insomnia. } \\
\text { More symptoms are } \\
\text { associated with shorter } \\
\text { sleep, especially with } \\
\text { inattentive symptoms. }\end{array}$ \\
\hline $\begin{array}{l}\text { ADHD-H vs ADHD-I: greater } \\
\text { insomnia severity }\end{array}$ & $\begin{array}{l}\text { SDB: higher SDB symptoms } \\
\text { are associated with higher } \\
\text { total ADHD symptoms. } \\
\text { This association is stronger } \\
\text { for hyperactivity, followed } \\
\text { by impulsivity. SDB and } \\
\text { inattentive symptoms were } \\
\text { not correlated. }\end{array}$ & $\begin{array}{l}\text { ADHD vs } \\
\text { normative } \\
\text { data: worse } \\
\text { psychological } \\
\text { well-being, self- } \\
\text { esteem, friends } \\
\text { and family } \\
\text { quality of life, } \\
\text { and daily/school } \\
\text { functioning. }\end{array}$ & $\begin{array}{l}\text { ADHD vs } \\
\text { normative } \\
\text { data: worse } \\
\text { physical } \\
\text { well-being }\end{array}$ & $\begin{array}{l}\text { ADHD was associated } \\
\text { with more SDB symptoms. } \\
\text { Hyperactive symptoms } \\
\text { were more strongly } \\
\text { associated with SDB and } \\
\text { insomnia than inattentive } \\
\text { symptoms. } \\
\text { ADHD was associated with } \\
\text { worse socioemotional and } \\
\text { physical well-being. }\end{array}$ \\
\hline $\begin{array}{l}\text { ADHD vs controls: more likely } \\
\text { to report any sleep problems, } \\
\text { loud snoring, breathing pauses } \\
\text { during sleep, cataplexy, short } \\
\text { sleep duration, daytime } \\
\text { sleepiness, use of hypnotics, } \\
\text { extreme evening types, restless } \\
\text { legs, or periodic limb movements. } \\
\text { Treated ADHD vs untreated } \\
\text { ADHD: less likely to report } \\
\text { sleep problems, cataplexy, and } \\
\text { restless legs. } \\
\text { ADHD-C and ADHD-H vs } \\
\text { ADHD-l: more likely to report } \\
\text { restless legs. }\end{array}$ & $\mathrm{N} / \mathrm{A}$ & $\mathrm{N} / \mathrm{A}$ & $\mathrm{N} / \mathrm{A}$ & $\begin{array}{l}\text { ADHD is associated } \\
\text { with greater reporting of } \\
\text { symptoms of narcolepsy, } \\
\text { CRSD, RLS/PLMD, OSA/ } \\
\text { SDB, and insomnia. } \\
\text { Treatment of ADHD is } \\
\text { associated with better } \\
\text { sleep and less symptoms of } \\
\text { narcolepsy and RLS. } \\
\text { Hyperactive symptoms of } \\
\text { ADHD are associated with } \\
\text { RLS. }\end{array}$ \\
\hline
\end{tabular}

(Continued) 
Table I (Continued)

\begin{tabular}{|l|l|l|l|}
\hline Author and year & Sample & Study design & Diagnoses \\
\hline $\begin{array}{l}\text { van der Heijden et } \\
\text { al }(2018)^{93}\end{array}$ & $\begin{array}{l}358 \text { children } \\
\text { Sex: } 212 \text { males, I46 females } \\
\text { Age (mean } \pm \text { SD), years: } \\
9.02 \pm 2.06\end{array}$ & Cross-sectional & $\begin{array}{l}\text { ADHD: DSM-IV } \\
\text { criteria } \\
\text { Sleep: none } \\
\text { Chronotype: } \\
\text { CCTQ }\end{array}$ \\
& & & \\
\hline
\end{tabular}

Abbreviations: 6-OH MS, 6-hydroxymelatoninsulfate; ABAS-II, Adaptive Behavior Assessment System, second edition; ADHD, attention-deficit/hyperactivity disorder; ADHD-C, ADHD combined subtype; ADHD-DC, ADHD Diagnostic Checklist; ADHD-I, ADHD inattentive subtype; ADHD-RS, ADHD Rating Scale; AHI, Apnea-Hypoapnea Index; ANT, Attention Network Task; ASD, autism spectrum disorder; ASRS, ADHD Self-Report Scale; AT, adenotonsillectomy; BASC-2: Behavior Assessment for Children, second children; BDI, Beck Depression Inventory; BIS, behavioral inhibition system; BMI, body mass index; CAARS, Conners' Adult ADHD Rating; CAARS-S:L, Conners' Adult ADHD Rating Scales - Self-report, Long version; CBCL, Child Behavior Checklist; CBT, core body temperature; CCTQ, Children's Chronotype Questionnaire; CDI, Children's Depression Inventory; CGI-P, Conner's Global Index, parent version; CHSQ, Children's Sleep Habits Questionnaire; CIDI, Composite International Diagnostic Interview; CPRS, Conner's Parent Rating Scale; CPRS-R, Conner's Parent Rating Scale, revised; CPT, Continuous Performance Test; CRSD, circadian rhythm sleep disorder; CSF, cerebrospinal fluid; CSHQ, Children Sleep Habits Questionnaire; CSHS, Children Sleep Hygiene Scale; CTRS, Conner's Teacher Rating Scale; DAS, Differential Ability Scale; DISC-IV, Diagnostic Interview Schedule for Children; DIVA 2.0, Diagnostic Interview for ADHD in Adults; DLMO, dim light melatonin onset; DSISD, Duke Diagnostic Interview for Sleep Disorders; DSM-IV, Diagnostic and Statistical Manual, fourth edition; DSM-IV-TR, Diagnostic and Statistical Manual, fourth edition, text revision; DSPS, delayed sleep phase syndrome; EDAH, evaluation of deficit of attention and hyperactivity; ESS, Epworth Sleepiness Scale; FBB-HKS, Symptom Checklist for Attention Deficit Hyperactivity Disorders; FSS, Fatigue Severity Scale; GSAQ, Global Sleep Assessment Questionnaire; HADS, Hospital Anxiety and Depression Scale; ICD-9, International Classification of Diseases, ninth revision; ICSD-2, International Classification of Sleep Disorders, second edition; ICSD-3, International Classification of Sleep Disorders, third edition; IED, intra-/extradimensional set shift; IRLSSG, International Restless Leg Syndrome Study Group; IQ, intelligence quotient; ISI: Insomnia Severity Scale; ISI-C, Insomnia Severity Index for Children; IVA-CPT, Integrated Visual and Auditory Continuous Performance Test; JSS, Jenkins Sleep Scale; KINDL, Questionnaire for Measuring Health-related Quality of Life in Children and Adolescents; K-SADS, Kiddie Schedule for Affective Disorders and Schizophrenia; K-SADS-PL, Kiddie Schedule for Affective Disorders and Schizophrenia, present and lifetime version; K-WISC-III, Korean Wechsler Intelligence Scale for Children, third edition; MCTQ, Munich Chronotype Questionnaire; MEQ, Morningness-Eveningness Questionnaire; MFFT-KC, Matching Familiar Figure Test for Korean Children; MOCl, Maudsley Obsessive Compulsive Index; MPH, methylphenidate; MSLT, multiple sleep latency test; MWT-B, multiple choice vocabulary test; NEPSY, A Developmental Neuropsychological Assessment; OCD, obsessive-compulsive disorder; OClr, Obsessive-Compulsive Index-revised; OSA, obstructive sleep apnea; OSA-18, Obstructive Sleep Apnea- I8; OSAHS, obstructive sleep apnea hypopnea syndrome; PDSS, Pediatric Daytime Sleepiness Scale; PedsQL, Pediatric Quality of Life Inventory; PLMD, periodic limb movement disorder; PLMI, periodic limb movement index; PLMS, periodic limb movement syndrome; PSG, polysomnography; PSQI, Pittsburgh Sleep Quality Index; REM, rapid eye movement; RLDI, Restless Leg Diagnosis Index; RLS, Restless Leg Syndrome; RME, rapid maxillary expansion; RWT, Regensburger Wortflussigkeits-Test; SCARED, screen for child anxiety related disorders; SCID-I, Structured Clinical Interview for DSM-IV Axis I Disorders; SDB, sleep-disordered breathing; SDQ, Strengths and Difficulties Questionnaire; SDSC, Sleep Disturbance Scale for Children; SF-36, Short form (36) Health Survey; SHQ, Sleep Health Questionnaire; SMFQ, Moods and Feelings Questionnaire; SNS, Swiss Narcolepsy Scale; SOC, Stockings of Cambridge; SRBD, Sleep Related Breathing Disorders questionnaire; SSS, Stanford Sleepiness Scale; STAI, State-Trait Anxiety Index; TCI, Temperament and Character Inventory; WISC-R, Wechsler Intelligence Scale for Children, revised; WURS-K, Wender-Utah Rating Scale, short form.

of apnea and hypopnea events per hour of sleep, in individuals with OSA with and without ADHD. ${ }^{74}$

\section{Subjective measures}

School-age children. N/A; Adolescents. N/A; Adults. Increased levels of self-reported loud snoring and breathing pauses during sleep in individuals with ADHD compared with healthy controls. ${ }^{77}$

\section{Daytime impairments}

\section{Cognitive impairments}

\section{Subjective measures}

Pre-school and school-age children. In children with SDB, significantly more symptoms of hyperactivity/inattention but not impulsivity were reported compared with healthy controls. ${ }^{67,78}$ Adolescents. N/A; Adults. Individuals with OSA symptoms had high levels of self-reported inattention and hyperactivity when compared with individuals without OSA symptoms. ${ }^{79}$

\section{Objective measures}

School-age children. Performance of children who reported frequent snoring (over three times per week) was poorer on tasks measuring attention, executive functions, language, intellectual functioning, and information processing when compared with healthy children who did not snore as frequently ${ }^{67}$ (less than two times per week). In addition, children with diagnosed OSA performed worse on measures of sustained attention compared with typically developing children. ${ }^{72}$ Adolescents. N/A; Adults. N/A.

\section{Emotional impairments}

Pre-school and school-age children. Children with OSA and ADHD had lower ratings on quality of life compared with individuals with OSA alone. ${ }^{80}$ Adolescents. N/A; Adults. Individuals with OSA and ADHD had lower ratings on quality of life and higher levels of reported symptoms of depression and anxiety than individuals with OSA alone. ${ }^{74}$ 


\begin{tabular}{|l|l|l|l|l|}
\hline Sleep differences & Daytime impairments & Emotional & Physical & \\
\hline & Cognitive & N/A & N/A & $\begin{array}{l}\text { Children with ADHD have } \\
\text { shorter sleep duration and } \\
\text { longer sleep latency. }\end{array}$ \\
\hline $\begin{array}{l}\text { Insomnia: ADHD vs controls: } \\
\text { shorter sleep duration and }\end{array}$ & N/A & & & \\
longer sleep onset latencies. & & & & \\
DSPS: no differences in & & & & \\
chronotype between ADHD, & & & & \\
ASD, and healthy children. & & & & \\
Eveningness predicted sleep & & & \\
problems in all three groups. & & & & \\
\hline
\end{tabular}

\section{Physical impairments}

Pre-school and school-age children. Severity of ADHD symptoms was associated with allergic rhinitis, adenoid hypertrophy, and tonsil hypertrophy in individuals with ADHD and OSA.$^{80}$ Adolescents. N/A; Adults. No difference was found in body mass index (BMI) between individuals with OSA and ADHD and OSA alone. ${ }^{74}$

\section{ADHD and RLS}

The present review includes seven studies (four crosssectional, two case-control, and one randomized control trial) that examined the sleep characteristics associated with RLS and ADHD.

\section{Sleep characteristics ${ }^{81-85}$ \\ PSG}

School-age children. A higher periodic limb movement disorder index was found for children with ADHD and RLS compared with healthy controls ${ }^{83}$ and for children with ADHD alone compared with healthy controls. ${ }^{82}$ Individuals with ADHD alone did not differ in periodicity of leg movements compared with healthy controls. ${ }^{82}$ Children with ADHD and RLS showed prolonged sleep latency, increased number of stage shifts, awakenings, and increased percentage of sleep stage 1 compared with healthy controls ${ }^{83}$ Adolescents. N/A; Adults. Individuals with ADHD had a higher periodic limb movement disorder index, longer sleep latency, and increased periodicity of leg movements compared with healthy controls. ${ }^{85}$

\section{Actigraphy}

School-age children. Multiple night awakenings, sleep hyperkinesias, and periodic limb movements were found in children with ADHD. ${ }^{62}$ Adolescents. N/A; Adults. N/A.

\section{Subjective measures}

School-age children. Children with ADHD reported more restless legs symptoms when compared with healthy controls. ${ }^{84}$ Adolescents. N/A; Adults. Higher levels of ADHD symptoms were reported by individuals with RLS, but the association between RLS and ADHD was no longer significant when accounting for sleep disturbances. ${ }^{86}$

\section{Physiological correlates}

School-age children. No differences were found in serum transferrin and iron levels between children with ADHD and healthy controls ${ }^{84}$ Regarding ferritin levels two studies ${ }^{84,87}$ reported no difference in ferritin levels between individuals with ADHD and healthy controls, whereas another study ${ }^{81}$ reported overall low ferritin levels in individuals with ADHD. Adolescents. N/A; Adults. No differences were found in serum ferritin and iron levels between individuals with RLS with or without ADHD. In addition, iron and ferritin levels did not correlate with RLS severity scores. ${ }^{87}$

\section{Cognitive impairments}

\section{Subjective measures}

School-age children. N/A; Adolescents. N/A; Adults. N/A.

\section{Objective measures}

School-age children. Verbal IQ on the Wechsler Intelligence Scale was positively correlated with stages 3 and 4 of sleep and limb movement index with arousals. It was negatively correlated with stage 2 of sleep. ${ }^{82}$ On the Matching Familiar Figure Test for Korean Children (MFFT-KC), an instrument designed to measure reflection-impulsivity, more response errors were associated with longer sleep time and limb movement index with arousals and longer reaction time was associated with shorter duration of stage 2 of sleep. ${ }^{82}$ Adolescents. N/A; Adults N/A. 


\section{Emotional impairments}

No studies were conducted to assess emotional outcomes in individuals with RLS and ADHD.

\section{Physical impairments}

School-age children. N/A; Adolescents. N/A; Adults. A study found that RLS participants were more likely to be obese (BMI >30) than participants without RLS (28.5\% of the RLS group compared with $10.1 \%$ of healthy controls). ${ }^{86}$

\section{ADHD and CRSD}

The present review includes eight studies (two cross-sectional, four case-control studies, and two open-label) that examined the sleep characteristics associated with CRSD and ADHD.

\section{Sleep characteristics}

PSG

No PSG study was conducted in individuals with ADHD and CRSD.

\section{Actigraphy}

School-age children. N/A; Adolescents. N/A; Adults. An actigraphic study revealed that sleep start time was 2:20 hours later, and sleep duration 1:08 hours shorter, sleep midpoint was delayed by 1 hour and 52 minutes, and sleep efficiency was higher in adolescents with ADHD and DSPS compared with healthy controls. ${ }^{69}$

\section{Objective circadian measures}

School-age children and adolescents. Children and adolescents with ADHD (6-16 years) had higher urinary levels of 6-hydoxymelatoninsulfate (a urine melatonin metabolite) at daytime, nighttime, and over 24 hours compared with healthy controls. ${ }^{88,89}$ Treatment with methylphenidate reduced urinary melatonin excretion and lowered morning melatonin in children with ADHD. ${ }^{90}$ Adults. Dim light melatonin onset occurred 1 hour and 23 minutes later in individuals with ADHD and DSPS compared with healthy controls..$^{91}$

\section{Subjective sleep and circadian measures}

School-age children. Inconsistent findings were found in children with ADHD manifesting greater eveningness in one of the two studies conducted to assess circadian tendencies in this population compared with healthy controls. ${ }^{92,93}$ Evening chronotype was associated with more resistance to sleep, respiratory problems, and daytime sleepiness..$^{92}$ Adolescents. Individuals with higher ADHD symptoms ( $>90$ th percentile on the ADHD Self-Report Scale [ASRS]) reported later bed and wake up times, shorter sleep duration, and lower sleep efficiency compared with individuals with low ADHD scores ( $<90$ th percentile on ASRS) ${ }^{94}$ Adults. Greater eveningness was found in individuals with ADHD compared with healthy controls. Eveningness was related to higher severity of the ADHD symptoms. ${ }^{40,95}$

\section{Daytime impairments Cognitive impairments}

Subjective measures

School-age children. N/A; Adolescents. N/A; Adults. Delayed sleep timing and daytime sleepiness were associated with higher levels of reported inattention and hyperactivity in participants with ADHD and DSPD compared with healthy controls. ${ }^{96}$ Evening chronotype was associated with higher levels of ADHD symptoms. ${ }^{40,89,95}$

\section{Objective measures}

No studies using objective cognitive measures were conducted in individuals with CRSD and ADHD.

\section{Emotional impairments}

No studies were conducted to assess emotional outcomes in individuals with CRSD and ADHD.

\section{Physical impairments}

School-age children. Psychosomatic symptoms (stomach aches, aches and pains, complains of headaches, seems tired) on the Conner's Parent Rating Scale were correlated to eveningness in the ADHD group. ${ }^{92}$ Adolescents. N/A; Adults. N/A.

\section{ADHD and insomnia}

The present review includes nine studies (three crosssectional, five case-control, and one randomized control trial) that examined the sleep characteristics associated with insomnia and ADHD.

\section{Sleep characteristics \\ PSG}

No PSG study was conducted in individuals with ADHD and insomnia.

\section{Actigraphy}

School-age children. Actigraphic studies ${ }^{97,98}$ revealed longer sleep onset latency, lower sleep efficiency, and lower total sleep time in children with ADHD alone compared with 
healthy controls. However, these measures were worse for children with ADHD and a comorbid psychiatric disorder (eg, anxious, depressed, oppositional symptoms) when compared with individuals with ADHD alone and healthy controls. ${ }^{97}$ Adolescents. N/A; Adults. N/A.

\section{Subjective sleep measures}

School-age children. Parental reports described longer sleep onset delay, ${ }^{97,99}$ shorter sleep duration, and more restless sleep in children with ADHD compared with healthy controls. ${ }^{97}$ Children with ADHD hyperactive/impulsive subtype scored higher on measures of insomnia compared with individuals with an inattentive type, ${ }^{100}$ and no differences in insomnia scores between children with ADHD and healthy controls were found in another study. ${ }^{99}$ Adolescents. Sleep duration and time in bed were shorter for individuals scoring high (over 90th percentile) in self-reported ADHD symptoms compared with those with low scores (<90th percentile). ${ }^{94}$ The association was stronger for individuals with ADHDinattentive subtype compared with ADHD hyperactive or combined subtypes. ${ }^{74}$ Adults. Subjective complaints of poor sleep quality, EDS sleep onset insomnia, and interrupted sleep as per the cutoff scores on the Epworth Sleepiness Scale and Pittsburgh Sleep Quality Index were prevalent in samples of individuals with ADHD compared with controls. ${ }^{47}$ Sleep quality was poorer in individuals with ADHD and an inattentive subtype compared with individuals with a combined subtype. ${ }^{101}$ Individuals with ADHD reported prolonged sleep latencies (>30 minutes) on the Pittsburgh Sleep Quality Index compared with controls. ${ }^{102}$ Adult women with ADHD reported higher prevalence of insomnia (43.9\% vs $12.2 \%$ ) compared with women without ADHD. ${ }^{103}$

\section{Daytime impairments}

\section{Cognitive impairments}

Subjective measures

No studies using subjective cognitive measures were conducted in individuals with insomnia and ADHD.

\section{Objective measures}

School-age children. N/A. Adolescents. Individuals with ADHD and shorter duration of nighttime sleep had more omission errors compared with healthy controls on a measure of executive function. ${ }^{99}$ Adults. N/A.

\section{Emotional impairments}

School-age children. Quality of life scores were reduced in children with insomnia and ADHD compared with healthy controls. ${ }^{100}$ Adolescents. N/A. Adults. N/A.

\section{Physical impairments}

School-age children. N/A. Adolescents. N/A; Adults. N/A.

\section{ADHD and narcolepsy}

The present review includes six studies (four cross-sectional and two case-control) that examined the sleep characteristics associated with narcolepsy and ADHD.

\section{Sleep and sleepiness \\ PSG}

School-age children. PSG studies showed longer total sleep times, shorter sleep latency, and shorter onset of REM periods in children with ADHD and narcolepsy compared with healthy controls. ${ }^{81,104}$ Adolescents and adults. Shorter stage 1 and longer stage 3 of sleep were found in adolescents and adults with narcolepsy and ADHD compared with individuals with narcolepsy without ADHD. ${ }^{57}$

\section{Actigraphy}

School-age children. Participants with ADHD and sleep problems, including three individuals with narcolepsy, displayed sleep efficiency of $<90 \% .{ }^{81}$ Adolescents. N/A; Adults. N/A.

Multiple Sleep Latency Test (a diagnostic tool used to objectively measure sleepiness and early-onset REM sleep) $)^{105}$

School-age children. N/A; Adolescents and Adults. Individuals with ADHD and comorbid narcolepsy type 2 had shorter REM sleep latencies compared with individuals with narcolepsy type 2 alone. $^{57}$

\section{Subjective measures}

Four studies used subjective measures to describe sleep outcomes (Children Sleep Habits Questionnaire, Pediatric Daytime Sleepiness Scale, Epworth Sleepiness Scale, Stanford Sleepiness Scale). For children, parents were the main respondents to the questionnaires. Clinicians also provided subjective information after interviewing the children.

School-age children. Higher levels of ADHD symptoms were associated with higher levels of reported sleepiness and fatigue ${ }^{81,106}$ Adolescents. N/A; Adults. Individuals with attention deficits and narcolepsy reported more sleepiness compared with healthy controls. ${ }^{107}$

\section{Cognitive impairments} Subjective measures

School-age children. Higher levels of inattention was found in children with narcolepsy compared with healthy controls. ${ }^{104,106}$ Poor sleep and fatigue in participants diagnosed 
with narcolepsy were associated with increased levels of inattention; ${ }^{106}$ Adolescents. N/A; Adults. Higher levels of inattention symptoms and higher hyperactivity scores were found in patients with narcolepsy compared with healthy controls. $^{108}$

\section{Objective measures}

School-age children. N/A; Adolescents. N/A; Adults. When comparing individuals with ADHD and narcolepsy to healthy controls, no significant differences were found in attention span and verbal working memory. ${ }^{107}$ However, reduced verbal fluency, ${ }^{107}$ greater difficulty in performing under time pressure, poorer performance on measures of executive functions (Go/no go test, ${ }^{107}$ Attention Network Test ${ }^{108}$ ) were observed in adults with narcolepsy and ADHD compared with healthy controls.

\section{Emotional impairments}

School-age children. Children with narcolepsy and comorbid ADHD symptoms had more depressive symptoms and decreased quality of life compared with healthy controls. ${ }^{106}$ Adolescents. N/A; Adults. Higher depression and ADHD scores were found in individuals with narcolepsy type 1 compared with controls. ${ }^{107,108}$

\section{Physical impairments}

School-age children. One study found a higher proportion of overweight or obesity in children with narcolepsy compared with healthy controls. ${ }^{106}$ Although the narcolepsy group did have a higher proportion of ADHD individuals and individuals with overweight and obesity, the association between ADHD and obesity was not examined. Adolescents. N/A; Adults. Higher BMI was found in individuals with ADHD and narcolepsy type 1 compared with participants with narcolepsy type 2 and healthy controls. ${ }^{108}$

\section{Discussion}

This review aimed to describe empirical studies pertaining to sleep abnormalities and outcomes in individuals with ADHD and comorbid primary sleep disorders in order to identify and address management challenges associated with treating these individuals.

Consistent with many previous studies, reviews, and meta-analyses, most of the PSG studies have failed in establishing consistent sleep differences in sleep architecture between individuals with sleep disorders with and without ADHD. However, a few differences have emerged. Children with ADHD and RLS were found to have an increased per- centage of sleep stage 1 compared with healthy controls, ${ }^{83}$ whereas for individuals with ADHD and narcolepsy, a shorter stage $1^{57}$ and shorter REM latency ${ }^{57,104}$ were found across all age groups compared with individuals without ADHD.

A few studies have also shown that children with ADHD and RLS and adults with ADHD alone have longer sleep latencies compared with healthy controls, ${ }^{83,85}$ and children with ADHD and narcolepsy have shorter sleep latencies compared with healthy controls. ${ }^{81,104}$ In addition, children with ADHD and narcolepsy were found to have lower sleep efficiency compared with healthy controls. ${ }^{81}$ In an actigraphic study, only children with ADHD were found to have more night awakenings and increased level of hyperkinesias. ${ }^{81}$

Subjective sleep reports both from parents of children with ADHD and adults with ADHD have consistently reported high prevalence of sleep problems. ${ }^{40,47,77,81,86,92,94,95,97,99,101,106,107}$ Higher level of reported sleepiness was found for individuals with ADHD and $\mathrm{OSA}^{77}$ and individuals with ADHD symptoms and narcolepsy ${ }^{81,106,107}$ compared with those without ADHD and healthy controls, respectively. Individuals with ADHD reported increased daytime sleepiness across studies $^{40,47,81,92,106,107}$ as well as shorter sleep duration ${ }^{82,91,94,97}$ compared with healthy controls. Finally, having an evening circadian type was more prevalent for individuals with $\mathrm{ADHD}^{40,89,92,95}$ compared with healthy controls.

Only a few empirical studies have examined daytime impairments in individuals with ADHD and comorbid primary sleep disorders. Regarding cognitive impairments, studies revealed higher levels of reported symptoms of hyperactivity and inattention in children with $\mathrm{SDB}^{68,78}$ and narcolepsy ${ }^{104,106}$ and adults with OSA, ${ }^{79}$ DSPD, ${ }^{96}$ and narcolepsy ${ }^{108}$ compared with healthy controls. Studies using objective cognitive measures revealed variable impairments across disorders and age groups. Children with OSA symptoms had impairments in attention, executive functions, language, intellectual functioning, and information processing compared with those without OSA symptoms. ${ }^{67,72}$ The impairment in executive functions and verbal fluency was also seen in adults with ADHD and narcolepsy ${ }^{108}$ compared with healthy controls. ${ }^{107}$ There were no empirical studies looking at cognitive impairments in individuals with ADHD and insomnia or RLS across the different age groups.

There is a dearth of empirical studies examining emotional impairments related to disrupted sleep in individuals with ADHD and primary sleep disorders. Lower perceived quality of life was reported in children with ADHD and SDB,${ }^{80}$ insomnia ${ }^{100}$ and narcolepsy, ${ }^{106}$ and adults with ADHD 
and $\mathrm{SDB}^{74}$ compared with healthy controls. Higher level of depression and anxiety symptoms were reported in adults with ADHD and $\mathrm{OSA}^{74}$ and narcolepsy ${ }^{107}$ alone compared with healthy controls. A complete lack of empirical data is noted for the assessment of emotional impairments in individuals with ADHD and RLS, CRDS, or adults with insomnia. Knowing that sleep disturbances significantly intensify emotional reactivity and negative mood and impair emotional regulation, this lack of information represents a significant gap in knowledge as well as a significant barrier to effective clinical management.

Scarce empirical data exist for physical impairments or comorbid conditions in individuals with ADHD and primary sleep disorders. For children with ADHD and SDB, an increase in medical comorbidities including allergic rhinitis, tonsillar, and adenoid hypertrophy was found compared with healthy controls $;^{80}$ however, this finding cannot be generalized across the age groups due to lack of empirical data. Furthermore, these medical comorbidities are unlikely to be present in adults since these structures (adenoids/ tonsils) atrophy following puberty. ${ }^{109}$ In addition to medical comorbidities, the presence of comorbid primary sleep disorders was associated with increased BMI. Adults with RLS $^{86}$ and narcolepsy ${ }^{108}$ are both found to have higher BMI compared with individuals without RLS and healthy controls, respectively, and children with narcolepsy have increased overweight and obesity compared with healthy controls. ${ }^{106}$ There are no empirical data regarding physical impairments or medical comorbidities for individuals with ADHD and insomnia across ages as well as children with ADHD and RLS and adults with ADHD and CRDS.

\section{Clinical implications}

The high prevalence of subjective complaints regarding sleep disturbances in individuals with ADHD across all age groups, and the prevalence of daytime symptoms of inattention and hyperactivity in individuals with primary sleep disorders, suggests that a baseline sleep evaluation during the initial assessment of ADHD as well as regular systematic screening for sleep problems is necessary component of ongoing ADHD management. This is of particular importance given that the presence of such sleep problems will likely decrease the efficacy of an intervention aimed solely at improving ADHD symptoms. As a first step, it is essential to delineate the nature of the reported sleep problems in order to eventually be able to properly treat them. It is also a possibility that there may be concomitant sleep disorders that are manifested simultaneously; therefore, having a thorough and systematic approach that includes objective sleep measures such as PSG, actigraphy, Multiple Sleep Latency Test along with obtaining detailed clinical history of the sleep, and ADHD challenges will help in establishing a clear diagnosis.

In order to undergo such tests, the treating physician will need to have access to referral to a facility that is capable of performing them. In turn, a sleep specialist will need access to a physician able to diagnose ADHD as well as psychological comorbidities in order to have a clear baseline. Collaboration between health professionals will be essential in treating these individuals and will be an integral part of the initial assessment.

Furthermore, a particular challenge will surface when assessing an adolescent population. Developmentally, during adolescence, sleep needs will naturally change. A key differentiation between normal sleep changes (eg, regular delayed sleep) and a sleep disorder (eg, DSPS) will need to be properly established.

Following a delineation of each problem, establishing a clear timeline of symptoms will be helpful to evaluate whether the symptoms of ADHD or the sleep disorder have a temporal relationship or whether they are comorbid. Moreover, it will be essential to establish whether the sleep problem is a cause or a consequence of the psychiatric comorbidity or vice versa and whether this stands true for the individual across their life span. Knowing that there is a significant prevalence of sleep disorders in ADHD and vice versa may lead to misattributions of symptoms of inattention and hyperactivity, for example, to ADHD and not a consequence of a sleep disorder. This issue will also impact the psychosocial treatment strategy as well as the pharmacologic treatment by a sleep specialist or a psychiatrist.

In addition to establishing a clear diagnosis, determining its impact or associations with daytime neuropsychological, emotional, or physical impairments would allow to map the sequence and magnitude of the required interventions. For example, if sleep disruption contributes to daytime inattention or executive dysfunction, treating it might be an ideal first step prior to or in addition to prescribing stimulant medications to address these challenges. Similarly, if sleep disturbances play a role in emotional dysregulation or low mood, treating sleep disturbance should be an important step in addressing the sleep and the comorbid disorders. In these cases, having a multidisciplinary approach will allow for simultaneous evaluation and treatment and collaboration with the goal of improving both the primary problem and the associated symptoms (eg, daytime impairments). 
In terms of treatment, the first step following assessment and diagnosis will consist of psychoeducation. Both the individual affected and the social entourage of these individuals (parents, spouses, etc) will need to have proper psychoeducation on ADHD symptoms as well as the particular sleep disorder in addition to its course, prognosis, treatment, and possible functional implications. Furthermore, education on normal sleep patterns and sleep hygiene will allow for nonpharmacological improvement of sleep.

Using medication to treat sleep disorders is widespread. Medication selection, particularly if treatment for ADHD is warranted, can be targeted to improve associated problems such as daytime impairments and should be integrated with behavioral strategies. For children with ADHD and SDB, surgical removal of the adenoids or tonsils is a first-line treatment, ${ }^{110}$ whereas for adults with ADHD and OSA, the use of positive airway pressure devices, oral appliances, or surgery are recommended treatment options. ${ }^{111}$ For individuals with ADHD and RLS, behavioral interventions could include modifying the sleep environment and treatment with iron supplementation ${ }^{112}$ (eg, ferrous sulfate) or gabapentin ${ }^{113}$ could be considered, in particular for a younger population. Treatment with dopaminergic agents such as pramipexole, ropinirole, L-DOPA, and a relatively new medication, rotigotine, in an adult population could also be an option. ${ }^{114}$ For individuals with ADHD and DSPS, treatment with light therapy ${ }^{115}$ and chronotherapy ${ }^{116}$ can be explored as well as using timed melatonin treatment. ${ }^{117}$ Treatment for individuals with ADHD and insomnia will vary according to age group, and in addition, a clear distinction between insomnia and DSPS will have to be made given that treatment for DSPS will be different than that for insomnia. For children with ADHD and insomnia, behavioral treatments such as positive reinforcement, scheduled awakenings, unmodified extinction, and faded bedtime could be starting options. ${ }^{118}$ For adolescents with ADHD and insomnia, cognitive behavioral therapy for insomnia (CBT-I) can be an effective treatment. ${ }^{119}$ In adults with insomnia, CBTI, stimulus control therapy, relaxation training, sleep restriction, multicomponent therapy, paradoxical intention, and biofeedback can be used alone or in conjunction with sleep medication, ${ }^{118}$ although treatment with pharmacotherapy for insomnia has weak evidence. ${ }^{120}$ For individuals with ADHD and narcolepsy, treatment with modafinil, sodium oxybate, or psychostimulants is indicated and it can be supplemented with education on sleep hygiene. ${ }^{121}$ Treating with psychostimulants would allow for the added benefit of treating both underlying disorders and not restrict the treating physician for the treatment of symptoms only.

\section{Limitations and future directions}

The limitations of available empirical evidence give rise to several challenges. A key challenge is integrating information from studies that differ in the participants included in the clinical and/or the control groups. Although all the studies included in the present work examined sleep and/or daytime impairments of individuals with ADHD and primary sleep disorders, some included only individuals with diagnosed ADHD in the study group, whereas others included individuals with sleep disorders only or individuals with a diagnosis of both ADHD and a primary sleep disorder. Studies also varied in the characteristics of the control group, with some comparing the study group with a different clinical group and others comparing the study group with healthy controls. In addition, some studies included individuals with psychological comorbidities or individuals undergoing pharmacotherapeutic treatment, making the sample more heterogeneous. The interstudy differences in the study and control groups limited the ability to generalize findings across studies in some cases that precluded direct comparison of studies. A second challenge in the present work was that, for children, many of studies used parent reports or clinician evaluations to obtain information on subjective sleep measures and daytime impairments. Due to the use of subjective and retrospective reports from parents, the results must be interpreted with caution. In adolescents, few studies assessed objective and subjective sleep impairments or daytime impairments, and overall there is a dearth of literature for this population. The present work was made even more challenging by the small number of longitudinal and experimental studies that have been published in this area. The lack of such studies limited the ability to establish a better understanding of the nature and the direction of the associations between primary sleep disorders and ADHD. An alarming shortage of empirical evidence on daytime impairments caused by sleep disturbances in individuals with ADHD was noted across all domains and all age groups. Furthermore, in the few studies to date that have examined daytime impairments, the cognitive, emotional, or physical outcomes were poorly defined and were inconsistent between studies. This significantly limited the ability of the review to ascertain the nature and magnitude of the impairments caused by the presence of a primary sleep disorder in individuals with ADHD, above and beyond or in combination with the impairments they present with as a result of their ADHD.

Future research should focus on the systematic and thorough evaluation of daytime impairments associated with the combined impacts of ADHD and sleep disturbances on indi- 
viduals with both conditions. Such research should clearly define outcomes that take into consideration the manners in which cognitive, emotional, and physical impairment may manifest at different ages, and should apply those definitions systematically across disorders. Ideally, these studies would compare individuals with ADHD and each of the primary sleep disorders known to be associated with ADHD to individuals with ADHD alone and to healthy controls. Results of these studies are needed to better appreciate patient needs and to inform clinical management.

Additional studies should also be conducted to capture the clinical characteristics and daytime impairments of all age groups, in particular adolescents and elderly patients (for whom there is no available empirical evidence). In addition, longitudinal studies are needed that evaluate changes in sleep, ADHD, and potential impairment over time, and that track the impacts of interventions on the sleep and daytime functioning of individuals with ADHD and comorbid sleep disorders.

\section{Disclosure}

The authors report no conflicts of interest in this work.

\section{References}

1. Faraone SV, Sergeant J, Gillberg C, Biederman J. The worldwide prevalence of ADHD: is it an American condition? World Psychiatry. 2003;2(2):104.

2. Faraone SV, Biederman J. What is the prevalence of adult ADHD? Results of a population screen of 966 adults. J Atten Disord. 2005;9(2):384-391.

3. American Psychiatric Association. Diagnostic and Statistical Manual of Mental Disorders (DSM-5®). Arlington: American Psychiatric Association Publishing; 2013.

4. Corkum P, Tannock R, Moldofsky H. Sleep disturbances in children with attention-deficit/hyperactivity disorder. J Am Acad Child Adolesc Psychiatry. 1998;37(6):637-646.

5. Gau SS, Kessler RC, Tseng WL, et al. Association between sleep problems and symptoms of attention-deficit/hyperactivity disorder in young adults. Sleep. 2007;30(2):195-201.

6. Fisher BC, Garges DM, Yoon SY, et al. Sex differences and the interaction of age and sleep issues in neuropsychological testing performance across the lifespan in an ADD/ADHD sample from the years 1989 to 2009. Psychol Rep. 2014;114(2):404-438.

7. Bogdan AR, Reeves KW, Kwjbsm R. Sleep duration in relation to attention deficit hyperactivity disorder in American adults. Behav Sleep Med. 2018;16(3):235-243.

8. Gruber R. Sleep characteristics of children and adolescents with attention deficit-hyperactivity disorder. Child Adolesc Psychiatr Clin NAm. 2009;18(4):863-876.

9. Konofal E, Lecendreux M, Cortese S. Sleep and ADHD. Sleep Med. 2010;11(7):652-658.

10. Weiss MD, Craig SG, Davies G, Schibuk L, Stein M. New research on the complex interaction of sleep and ADHD. Curr Sleep Med Rep. 2015;1(2):114-121.

11. Morse A, Sanjeev K. Narcolepsy and psychiatric disorders: comorbidities or shared pathophysiology? Med Sci. 2018;6(1):16.
12. Marcus C, Annett R, Brooks L. Cardiorespiratory sleep studies in children. Establishment of normative data and polysomnographic predictors of morbidity. American Thoracic Society. Am J Respir Crit Care Med. 1999;160(4):1381-1387.

13. Brouillette RT, Fernbach SK, Hunt CE. Obstructive sleep apnea in infants and children. J Pediatr. 1982;100(1):31-40.

14. Guilleminault C, Korobkin R, Winkle R. A review of 50 children with obstructive sleep apnea syndrome. Lung. 1981;159(5):275-287.

15. Constantin E, Low NC, Dugas E, Karp I, O’Loughlin J. Association between childhood sleep-disordered breathing and disruptive behavior disorders in childhood and adolescence. Behav Sleep Med. 2015;13(6):442-454.

16. Johnson EO, Roth T. An epidemiologic study of sleep-disordered breathing symptoms among adolescents. Sleep. 2006;29(9): 1135-1142.

17. Sedky K, Nazir R, Carvalho KS, Lippmann S. Attention deficit hyperactivity disorder and sleep disordered breathing in children. $J$ Pediatr Biochem. 2013;3(2):61-67.

18. Wei JL, Bond J, Mayo MS, Smith HJ, Reese M, Weatherly RA. Improved behavior and sleep after adenotonsillectomy in children with sleepdisordered breathing: long-term follow-up. Arch Otolaryngol Head Neck Surg. 2009;135(7):642-646.

19. Dillon JE, Blunden S, Ruzicka DL, et al. DSM-IV diagnoses and obstructive sleep apnea in children before and 1 year after adenotonsillectomy. JAm Acad Child Adolesc Psychiatry. 2007;46(11):1425-1436.

20. O'Brien LM, Holbrook CR, Mervis CB, et al. Sleep and neurobehavioral characteristics of 5- to 7-year-old children with parentally reported symptoms of attention-deficit/hyperactivity disorder. Pediatrics. 2003;111(3):554-563.

21. Sangal RB, Owens JA, Sangal J. Patients with attention-deficit/ hyperactivity disorder without observed apneic episodes in sleep or daytime sleepiness have normal sleep on polysomnography. Sleep. 2005;28(9):1143-1148.

22. Lal C, Strange C, Bachman D. Neurocognitive impairment in obstructive sleep apnea. Chest. 2012;141(6):1601-1610.

23. Beebe DW, Gozal D. Obstructive sleep apnea and the prefrontal cortex: towards a comprehensive model linking nocturnal upper airway obstruction to daytime cognitive and behavioral deficits. J Sleep Res. 2002;11(1):1-16.

24. Červenka S, Pålhagen SE, Comley RA, et al. Support for dopaminergic hypoactivity in restless legs syndrome: a PET study on D2-receptor binding. Brain. 2006;129(Pt 8):2017-2028.

25. Philipsen A, Hornyak M, Riemann D. Sleep and sleep disorders in adults with attention deficit/hyperactivity disorder. Sleep Med Rev. 2006;10(6):399-405.

26. Trenkwalder C, Paulus W, Walters AS. The restless legs syndrome. Lancet Neurol. 2005;4(8):465-475.

27. Wagner ML, Walters AS, Fisher BC. Symptoms of attention-deficit/ hyperactivity disorder in adults with restless legs syndrome. Sleep. 2004;27(8):1499-1504.

28. Cortese S, Angriman M, Lecendreux M, Konofal E. Iron and attention deficit/hyperactivity disorder: what is the empirical evidence so far? A systematic review of the literature. Expert Rev Neurother. 2012;12(10):1227-1240.

29. Cortese S, Azoulay R, Castellanos FX, et al. Brain iron levels in attention-deficit/hyperactivity disorder: a pilot MRI study. World J Biol Psychiatry. 2012;13(3):223-231.

30. Picchietti MA, Picchietti DL. Advances in pediatric restless legs syndrome: iron, genetics, diagnosis and treatment. Sleep Med. 2010;11(7):643-651.

31. Yeh P, Walters AS, Tsuang JW. Restless legs syndrome: a comprehensive overview on its epidemiology, risk factors, and treatment. Sleep Breath. 2012;16(4):987-1007.

32. Cortese S, Konofal E, Lecendreux M, et al. Restless legs syndrome and attention-deficit/hyperactivity disorder: a review of the literature. Sleep. 2005;28(8):1007-1013. 
33. Lewin DS, Pinto MD. Sleep Disorders and ADHD: Shared and Common Phenotypes. Sleep. 2004;27(2):188-189.

34. Picchietti D, Allen RP, Walters AS, Davidson JE, Myers A, Ferini-Strambi L. Restless legs syndrome: prevalence and impact in children and adolescents--the Peds REST study. Pediatrics. 2007;120(2):253-266.

35. Walters AS, Silvestri R, Zucconi M, Chandrashekariah R, Konofal E. Review of the possible relationship and hypothetical links between attention deficit hyperactivity disorder (ADHD) and the simple sleep related movement disorders, parasomnias, hypersomnias, and circadian rhythm disorders. J Clin Sleep Med. 2008;4(6):591-600.

36. Wagner ML, Walters AS, Fisher BC. Symptoms of attention-deficit/ hyperactivity disorder in adults with restless legs syndrome. Sleep. 2004;27(8):1499-1504.

37. Konofal E, Cortese S, Marchand M, Mouren MC, Arnulf I, Lecendreux $\mathrm{M}$. Impact of restless legs syndrome and iron deficiency on attention-deficit/hyperactivity disorder in children. Sleep Med. 2007;8(7-8):711-715.

38. Cortese S, Lecendreux M, Mouren M-C, Konofal E. ADHD and insomnia. J Am Acad Child Adolesc Psychiatry. 2006;4(45):384-385.

39. Baird AL, Coogan AN, Siddiqui A, Donev RM, Thome J. Adult attention-deficit hyperactivity disorder is associated with alterations in circadian rhythms at the behavioural, endocrine and molecular levels. Mol Psychiatry. 2012;17(10):988-995.

40. Bron TI, Bijlenga D, Kooij JJ, et al. Attention-deficit hyperactivity disorder symptoms add risk to circadian rhythm sleep problems in depression and anxiety. J Affect Disord. 2016;200:74-81.

41. Kooij JJ, Bijlenga D. The circadian rhythm in adult attention-deficit/ hyperactivity disorder: current state of affairs. Expert Rev Neurother. 2013;13(10):1107-1116.

42. Sivertsen B, Harvey AG, Pallesen S, Hysing M. Mental health problems in adolescents with delayed sleep phase: results from a large populationbased study in Norway. J Sleep Res. 2015;24(1):11-18.

43. Gruber R, Fontil L, Bergmame L, et al. Contributions of circadian tendencies and behavioral problems to sleep onset problems of children with ADHD. BMC Psychiatry. 2012;12(1):212.

44. van der Heijden KB, Smits MG, van Someren EJ, Gunning WB. Idiopathic chronic sleep onset insomnia in attention-deficit/hyperactivity disorder: a circadian rhythm sleep disorder. Chronobiol Int. 2005;22(3):559-570.

45. American Academy of Sleep Medicine. International Classification of Sleep Disorders-Third Edition (ICSD-3). Darien, IL: American Academy of Sleep Medicine; 2014.

46. Sung V, Hiscock H, Sciberras E, Efron D. Sleep problems in children with attention-deficit/hyperactivity disorder: prevalence and the effect on the child and family. Arch Pediatr Adolesc Med. 2008;162(4):336-342.

47. Brevik EJ, Lundervold AJ, Halmøy A, et al. Prevalence and clinical correlates of insomnia in adults with attention-deficit hyperactivity disorder. Acta Psychiatr Scand. 2017;136(2):220-227.

48. Calhoun SL, Fernandez-Mendoza J, Vgontzas AN, Liao D, Bixler EO. Prevalence of insomnia symptoms in a general population sample of young children and preadolescents: gender effects. Sleep Med. 2014;15(1):91-95.

49. Nowicki Z, Grabowski K, Cubała WJ, et al. Prevalence of selfreported insomnia in general population of Poland. Psychiatr Pol. 2016;50(1):165-173.

50. Ohayon MM. Epidemiology of insomnia: what we know and what we still need to learn. Sleep Med Rev. 2002;6(2):97-111.

51. Weiss MD, Wasdell MB, Bomben MM, Rea KJ, Freeman RD. Sleep hygiene and melatonin treatment for children and adolescents with ADHD and initial insomnia. $J$ Am Acad Child Adolesc Psychiatry. 2006;45(5):512-519.

52. Ohayon MM. Narcolepsy is complicated by high medical and psychiatric comorbidities: a comparison with the general population. Sleep Med. 2013;14(6):488-492.

53. Cortese S, Konofal E, Lecendreux M. Alertness and feeding behaviors in ADHD: does the hypocretin/orexin system play a role? Med Hypotheses. 2008;71(5):770-775.
54. Oosterloo M, Lammers GJ, Overeem S, de Noord I, Kooij JJ. Possible confusion between primary hypersomnia and adult attention-deficit/ hyperactivity disorder. Psychiatry Res. 2006;143(2-3):293-297.

55. Stores G, Montgomery P, Wiggs L. The psychosocial problems of children with narcolepsy and those with excessive daytime sleepiness of uncertain origin. Pediatrics. 2006;118(4):e1116-e1123.

56. Nordstrand SH, Hansen BH, Kamaleri Y, Nilsen KB, Rootwelt T, Karlsen TI, Knudsen S. Changes in quality of life in individuals with narcolepsy type 1 after the H1N1-influenza epidemic and vaccination campaign in Norway: a two-year prospective cohort study. Sleep Med. 2018;50:175-180

57. Ito W, Honda M, Ueno T, Kato N. Hypersomnia with ADHD: a possible subtype of narcolepsy type 2. Sleep Biol Rhythms. 2018;16(2):205-210.

58. del Campo N, Chamberlain SR, Sahakian BJ, Robbins TW. The roles of dopamine and noradrenaline in the pathophysiology and treatment of attention-deficit/hyperactivity disorder. Biol Psychiatry. 2011;69(12):e145-e157.

59. Jouvet $\mathrm{M}$. The role of monoamines and acetylcholine-containing neurons in the regulation of the sleep-waking cycle. Ergeb Physiol. 1972;64:166-307.

60. Cherkasova MV, Faridi N, Casey KF, et al. Amphetamine-induced dopamine release and neurocognitive function in treatment-naive adults with ADHD. Neuropsychopharmacology. 2014;39(6):1498-1507.

61. Reddy DS. Current pharmacotherapy of attention deficit hyperactivity disorder. Drugs Today. 2013;49(10):647-665.

62. de La Herrán-Arita AK, García-García F. Current and emerging options for the drug treatment of narcolepsy. Drugs. 2013;73(16):1771-1781.

63. Instanes JT, Klungsøyr K, Halmøy A, Fasmer OB, Haavik J. Adult ADHD and comorbid somatic disease: a systematic literature review. J Atten Disord. 2018;22(3):203-228.

64. Díaz-Román A, Mitchell R, Cortese S. Sleep in adults with ADHD: systematic review and meta-analysis of subjective and objective studies. Neurosci Biobehav Rev. 2018;89:61-71.

65. Coogan AN, Mcgowan NM. A systematic review of circadian function, chronotype and chronotherapy in attention deficit hyperactivity disorder. Atten Defic Hyperact Disord. 2017;9(3):129-147.

66. Lunsford-Avery JR, Krystal AD, Kollins SH. Sleep disturbances in adolescents with ADHD: a systematic review and framework for future research. Clin Psychol Rev. 2016;50:159-174.

67. Smith DL, Gozal D, Hunter SJ, Kheirandish-Gozal L. Frequency of snoring, rather than apnea-hypopnea index, predicts both cognitive and behavioral problems in young children. Sleep Med. 2017;34:170-178.

68. Kim JY, Lee CH, Kim H-M. Behavioral consequences of children with sleep-disordered breathing after adenotonsillectomy. World J Pediatr. 2018;14(1):57-65.

69. Smith DL, Gozal D, Hunter SJ, Philby MF, Kaylegian J, KheirandishGozal L. Impact of sleep disordered breathing on behaviour among elementary school-aged children: a cross-sectional analysis of a large community-based sample. Eur Respir J. 2016;48(6):1631-1639.

70. Smith DL, Gozal D, Hunter SJ, Kheirandish-Gozal L. Parent-reported behavioral and psychiatric problems mediate the relationship between sleep-disordered breathing and cognitive deficits in school-aged children. Front Neurol. 2017;8:410.

71. Villa MP, Sujanska A, Vitelli O, et al. Use of the sleep clinical record in the follow-up of children with obstructive sleep apnea (OSA) after treatment. Sleep Breath. 2016;20(1):321-329.

72. Zhu J, Fang Y, Chen X, et al. The impacts of obstructive sleep apnea hypopnea syndrome severity and surgery intervention on psychological and behavioral abnormalities and postoperative recovery in pediatric patients. Med Sci Monit. 2014;20:1474-1480.

73. Vitelli O, Tabarrini A, Miano S, et al. Impact of obesity on cognitive outcome in children with sleep-disordered breathing. Sleep Med. 2015;16(5):625-630.

74. Oğuztürk Ö, Ekici M, Çimen D, Ekici A, Senturk E. Attention deficit/ hyperactivity disorder in adults with sleep apnea. J Clin Psychol Med Settings. 2013;20(2):234-239. 
75. Perfect MM, Archbold K, Goodwin JL, Levine-Donnerstein D, Quan SF. Risk of behavioral and adaptive functioning difficulties in youth with previous and current sleep disordered breathing. Sleep. 2013;36(4):517-525.

76. Ekici A, Ekici M, Oğuztürk O, Karaboğa I, Çimen D, Senturk E. Personality profiles in patients with obstructive sleep apnea. Sleep Breath. 2013;17(1):305-310.

77. Bjorvatn B, Brevik EJ, Lundervold AJ, et al. Adults with attention deficit hyperactivity disorder report high symptom levels of troubled sleep, restless legs, and cataplexy. Front Psychol. 2017;8(1621):1621.

78. Amiri S, Abdollahifakhim S, Lotfi A, Bayazian G, Sohrabpour M, Hemmatjoo T. Effect of adenotonsillectomy on ADHD symptoms of children with adenotonsillar hypertrophy and sleep disordered breathing. Int J Pediatr Otorhinolaryngol. 2015;79(8):1213-1217.

79. Vogel SWN, Bijlenga D, Benjamins JS, Beekman ATF, Kooij JJS, van Someren EJW. Attention deficit hyperactivity disorder symptom severity and sleep problems in adult participants of the Netherlands sleep registry. Sleep Med. 2017;40:94-102.

80. Wu J, Gu M, Chen S, et al. Factors related to pediatric obstructive sleep apnea-hypopnea syndrome in children with attention deficit hyperactivity disorder in different age groups. Medicine. 2017;96(42): e8281.

81. Miano S, Esposito M, Foderaro G, Ramelli GP, Pezzoli V, Manconi M. Sleep-related disorders in children with attention-deficit hyperactivity disorder: preliminary results of a full sleep assessment study. CNS Neurosci Ther. 2016;22(11):906-914.

82. Um YH, Jeong JH, Hong SC, et al. Association between sleep parameters and cognitive function in drug-naïve children with attentiondeficit hyperactivity disorder: a polysomnographic study. Sleep Med 2016;21:165-170.

83. Ferri R, Bruni O, Novelli L, Picchietti MA, Picchietti DL. Time structure of leg movement activity during sleep in attention-deficit/hyperactivity disorder and effects of levodopa. Sleep Med. 2013;14(4):359-366.

84. Akinci G, Oztura I, Hiz S, et al. Sleep structure in children with attention-deficit/hyperactivity disorder. J Child Neurol. 2015;30(11): $1520-1525$.

85. Garbazza C, Sauter C, Paul J, et al. Leg movement activity during sleep in adults with attention-deficit/hyperactivity disorder. Front Psychiatry. 2018;9:179.

86. Roy M, de Zwaan M, Tuin I, Philipsen A, Brähler E, Müller A. Association between restless legs syndrome and adult ADHD in a German community-based sample. J Atten Disord. 2018;22(3):300-308.

87. Ghorayeb I, Gamas A, Mazurie Z, Mayo W. Attention-deficit hyperactivity and obsessive-compulsive symptoms in adult patients with primary restless legs syndrome: different phenotypes of the same disease? Behav Sleep Med. 2017;32(8):1-8.

88. Büber A, Çakaloz B, Işıldar Y, et al. Increased urinary 6-hydroxymelatoninsulfate levels in attention deficit hyperactivity disorder diagnosed children and adolescent. Neurosci Lett. 2016;617:195-200.

89. Molina-Carballo A, Naranjo-Gómez A, Uberos J, et al. Methylphenidate effects on blood serotonin and melatonin levels may help to synchronise biological rhythms in children with ADHD. J Psychiatr Res. 2013;47(3):377-383.

90. Snitselaar MA, Smits MG, van der Heijden KB, Smit A, Spijker J. Influence of methylphenidate on circadian rhythmicity and sleep in adult attention-deficit/hyperactivity disorder. Sleep Biol Rhythms. 2013;11(4):282-285.

91. Bijlenga D, van Someren EJ, Gruber R, et al. Body temperature, activity and melatonin profiles in adults with attention-deficit/hyperactivity disorder and delayed sleep: a case-control study. J Sleep Res. 2013;22(6):607-616.

92. Durmuş FB, Arman AR, Ayaz AB. Chronotype and its relationship with sleep disorders in children with attention deficit hyperactivity disorder. Chronobiol Int. 2017;34(7):886-894.

93. van der Heijden KB, Stoffelsen RJ, Popma A, Swaab H, Sleep SH. Sleep, chronotype, and sleep hygiene in children with attention-deficit/ hyperactivity disorder, autism spectrum disorder, and controls. Eur Child Adolesc Psychiatry. 2018;27(1):99-111.
94. Hysing M, Lundervold AJ, Posserud MB, Sivertsen B. Association between sleep problems and symptoms of attention deficit hyperactivity disorder in adolescence: results from a large population-based study. Behav Sleep Med. 2016;14(5):550-564.

95. Bumb JM, Mier D, Noelte I, et al. Associations of pineal volume, chronotype and symptom severity in adults with attention deficit hyperactivity disorder and healthy controls. Eur Neuropsychopharmacol. 2016;26(7):1119-1126.

96. Gamble KL, May RS, Besing RC, Tankersly AP, Fargason RE. Delayed sleep timing and symptoms in adults with attention-deficit/ hyperactivity disorder: a controlled actigraphy study. Chronobiol Int. 2013;30(4):598-606

97. Moreau V, Rouleau N, Morin CM. Sleep of children with attention deficit hyperactivity disorder: actigraphic and parental reports. Behav Sleep Med. 2014;12(1):69-83.

98. Corkum P, Lingley-Pottie P, Davidson F, et al. Better nights/better days-distance intervention for insomnia in school-aged children with/ without ADHD: a randomized controlled trial. J Pediatr Psychol. 2016;41(6):701-713.

99. Vélez-Galarraga R, Guillén-Grima F, Crespo-Eguílaz N, SánchezCarpintero R. Prevalence of sleep disorders and their relationship with core symptoms of inattention and hyperactivity in children with attention-deficit/hyperactivity disorder. Eur J Paediatr Neurol. 2016;20(6):925-937.

100. Grünwald J, Schlarb AA. Relationship between subtypes and symptoms of ADHD, insomnia, and nightmares in connection with quality of life in children. Neuropsychiatr Dis Treat. 2017;13:2341-2350.

101. Yoon SY, Jain UR, Shapiro CM. Sleep and daytime function in adults with attention-deficit/hyperactivity disorder: subtype differences. Sleep Med. 2013;14(7):648-655.

102. Fargason RE, Hollar AF, White S, Gamble KL. Adults with ADHDwithout insomnia history have subclinical sleep disturbance but not circadian delay: an ADHD phenotype? J Atten Disord. 2013;17(7): 583-588

103. Fuller-Thomson E, Lewis DA, Agbeyaka SK. Attention-deficit/hyperactivity disorder casts a long shadow: findings from a population-based study of adult women with self-reported ADHD. Child Care Health Dev. 2016;42(6):918-927.

104. Rocca FL, Finotti E, Pizza F, et al. Psychosocial profile and quality of life in children with type 1 narcolepsy: a case-control study. Sleep. 2016;39(7):1389-1398.

105. Thomas D, Anderson WM. Multiple Sleep Latency Test (MSLT). In: Kushida CA, editor. Encyclopedia of Sleep. Waltham: Academic Press; 2013:96-99.

106. Lecendreux M, Lavault S, Lopez R, et al. Attention-deficit/hyperactivity disorder (ADHD) symptoms in pediatric narcolepsy: a crosssectional study. Sleep. 2015;38(8):1285-1295.

107. Zamarian L, Högl B, Delazer M, et al. Subjective deficits of attention, cognition and depression in patients with narcolepsy. Sleep Med. 2015;16(1):45-51.

108. Filardi M, Pizza F, Tonetti L, Antelmi E, Natale V, Plazzi G. Attention impairments and ADHD symptoms in adult narcoleptic patients with and without hypocretin deficiency. PLoS One. 2017;12(8):e0182085.

109. Robertson CM. Anatomy and physiology of the tonsil. JAm Med Assoc 1909;LIII(9):684-689.

110. Marcus CL, Brooks LJ, Ward SD, et al. Diagnosis and management of childhood obstructive sleep apnea syndrome. Pediatrics. 2012;130(3):e714-e755.

111. Epstein LJ, Kristo D, Strollo PJ, et al. Clinical guideline for the evaluation, management and long-term care of obstructive sleep apnea in adults. J Clin Sleep Med. 2009;5(3):263-276.

112. Mohri I, Kato-Nishimura K, Kagitani-Shimono K, et al. Evaluation of oral iron treatment in pediatric restless legs syndrome (RLS). Sleep Med. 2012;13(4):429-432.

113. Garcia-Borreguero D, Larrosa O, de La Llave Y, Verger K, Masramon $\mathrm{X}$, Hernandez G. Treatment of restless legs syndrome with gabapentin: a double-blind, cross-over study. Neurology. 2002;59(10): $1573-1579$. 
114. Aurora RN, Kristo DA, Bista SR, et al. The treatment of restless legs syndrome and periodic limb movement disorder in adults--an update for 2012: practice parameters with an evidence-based systematic review and meta-analyses: an American Academy of Sleep Medicine Clinical Practice Guideline. Sleep. 2012;35(8):1039-1062.

115. Lack L, Wright H, Kemp K, Gibbon S. The treatment of earlymorning awakening insomnia with 2 evenings of bright light. Sleep. 2005;28(5):616-623.

116. Weitzman ED, Czeisler CA, Coleman RM, et al. Delayed sleep phase syndrome. A chronobiological disorder with sleep-onset insomnia. Arch Gen Psychiatry. 1981;38(7):737-746.

117. Auger RR, Burgess HJ, Emens JS, Deriy LV, Thomas SM, Sharkey KM. Clinical practice guideline for the treatment of intrinsic circadian rhythm sleep-wake disorders: Advanced Sleep-Wake Phase Disorder (ASWPD), Delayed Sleep-Wake Phase Disorder (DSWPD), Non24-Hour Sleep-Wake Rhythm Disorder (N24SWD), and Irregular Sleep-Wake Rhythm Disorder (ISWRD). An Update for 2015: An American Academy of Sleep Medicine Clinical Practice Guideline. $J$ Clin Sleep Med. 2015;11(10):1199-1236.
118. Morgenthaler T, Owens J, Alessi C, Boehlecke B, Brown T, Coleman Jr J. American Academy of Sleep Medicine Report. Practice parameters for behavioral treatment of bedtime problems and night wakings in infants and young children. Sleep. 2006;29: 1277-1281.

119. Gradisar M, Dohnt H, Gardner G, et al. A randomized controlled trial of cognitive-behavior therapy plus bright light therapy for adolescent delayed sleep phase disorder. Sleep. 2011;34(12):1671-1680.

120. Sateia MJ, Buysse DJ, Krystal AD, Neubauer DN, Heald JL. Clinical practice guideline for the pharmacologic treatment of chronic insomnia in adults: an American Academy of Sleep Medicine clinical practice guideline. J Clin Sleep Med. 2017;13(2):307-349.

121. Wise MS, Arand DL, Auger RR, Brooks SN, Watson NF, American Academy of Sleep Medicine. Treatment of narcolepsy and other hypersomnias of central origin. Sleep. 2007;30(12):1712-1727.

\section{Publish your work in this journal}

Nature and Science of Sleep is an international, peer-reviewed, open access journal covering all aspects of sleep science and sleep medicine, including the neurophysiology and functions of sleep, the genetics of sleep, sleep and society, biological rhythms, dreaming, sleep disorders and therapy, and strategies to optimize healthy sleep. The manuscript

\section{Dovepress}

management system is completely online and includes a very quick and fair peer-review system, which is all easy to use. Visit http://www. dovepress.com/testimonials.php to read real quotes from published authors. 